\title{
The triterpenoid CDDO-imidazolide ameliorates mouse liver ischemia-reperfusion injury through activating the Nrf2/HO-1 pathway enhanced autophagy
}

\author{
Dongwei $\mathrm{Xu}^{1,5}$, Lili Chen ${ }^{1,2,5}$, Xiaosong Chen ${ }^{1}$, Yankai Wen ${ }^{1,2}$, Chang $\mathrm{Yu}^{1,2}$, Jufang Yao ${ }^{3}$, Hailong $\mathrm{Wu}^{4}$, Xin Wang ${ }^{\star, 1}$, Qiang Xia ${ }^{\star, 1}$ and \\ Xiaoni Kong ${ }^{*, 1}$
}

Nuclear factor erythroid 2-related factor 2 (Nrf2)-mediated induction of antioxidants has been implicated to have protective roles in ischemia-reperfusion (I/R) injury in many animal models. However, the in vivo effects of CDDO-imidazole (CDDO-Im) (1-[2-cyano3-,12-dioxooleana-1,9(11)-dien-28-oyl] imidazole), a Nrf2 activator, in hepatic I/R injury is lacking and its exact molecular mechanisms are still not very clear. The goals of this study were to determine whether CDDO-Im can prevent liver injury induced by $\mathrm{I} / \mathrm{R}$ in the mouse, and to elucidate the molecular target of drug action. Mice were randomly equally divided into two groups and administered intraperitoneally with either DMSO control or CDDO-Im $(2 \mathrm{mg} / \mathrm{kg}) 3 \mathrm{~h}$ before subjected to $90-\mathrm{min}$ hepatic $70 \%$ ischemia followed by reperfusion. Subsequently, the Liver and blood samples of these mice were collected to evaluate liver injury. CDDO-Im pretreatment markedly improve hepatic I/R injury by attenuating hepatic necrosis and apoptosis, reducing reactive oxygen species (ROS) levels and inflammatory responses, and ameliorating mitochondrial dysfunction. Mechanistically, by using Nrf2 Knockout mice and hemeoxygenase 1 (HO-1) inhibitor, we found that these CDDO-Im protection effects are attributed to enhanced autophagy, which is mediated by activating Nrf2/HO-1 pathway. By accelerating autophagy and clearance of damaged mitochondria, CDDO-Im reduced the mtDNA release and ROS overproduction, and in turn decreased damage-associated molecular patterns induced inflammatory responses and the following secondary liver injury. These results indicate that by enhancing autophagy, CDDO-Im-mediated activation of Nrf2/HO-1 signaling could be a novel therapeutic strategy to minimize the adverse effects of hepatic I/R injury.

Cell Death and Disease (2017) 8, e2983; doi:10.1038/cddis.2017.386; published online 10 August 2017

Ischemia-reperfusion (I/R) injury widely exists in clinical procedures such as liver resection, organ transplantation, trauma and shock. Hepatic I/R injury remains an important issue affecting the long-term graft survival of patients with liver transplantation, and accounts for $\sim 10 \%$ of early postoperative liver failure and at least $10 \%$ of the acute or chronic rejection. ${ }^{1,2}$ However, there are still no effective drugs to protect the liver from $\mathrm{I} / \mathrm{R}$ injury so far. During the ischemic process, the absence of oxygen supplement and the consumption of glycogen lead to ATP metabolic disorder, mitochondrial dysfunction and initial of cell death. During the reperfusion process, activation of the Kupffer cells and neutrophils result in the production of lots of inflammatory cytokines and reactive oxygen species (ROS), leading to enhanced liver injury. ${ }^{3}$

Nuclear factor erythroid 2-related factor 2 (Nrf2) is a master transcription factor in regulating antioxidant production to maintain cellular redox homeostasis. ${ }^{4-6}$ Under normal circumstances, Nrf2 is located in the cytoplasm and degraded by its repressor Kelch-like erythroid-associated protein 1 (Keap1) through the ubiquitination and proteasome pathway. Although in pathological conditions, the interaction between Keap1 and Nrf2 is disrupted by numerous stimuli and activated Nrf2 is translocated into the nucleus to promote the transcription of many relevant cytoprotective and antioxidant genes such as $\mathrm{NAD}(\mathrm{P}) \mathrm{H}$ quinone dehydrogenase 1 and hemeoxygenase 1 $(\mathrm{HO}-1)$ via binding to the antioxidant response elements (AREs) in promoter regions of those genes. ${ }^{4}$ Previous studies have demonstrated that Nrf2 deficiency donor liver exacerbated IR injury in transplant recipients, whereas activation of Nrf2 has hepatoprotective effects. ${ }^{7-9}$ Furthermore, pharmacologic activation of Nrf2 has demonstrated to increase hepatic GSH concentrations, and therefore increase bile flow, which may be able to counteract cholestatic conditions and modify oxidative stress and inflammation in these diseases progression. ${ }^{9-11}$ CDDO-imidazolide (CDDO-Im), one of the synthetic oleanane triterpenoids, is a potent activator of the Nrf2 pathway and proved to be a protective agent in a number of disease models, including acute kidney, lung or neurons injury, acetaminophen hepatotoxicity, emphysema and sepsis. $^{12-17}$ Here, we hypothesized that CDDO-Im may protect hepatic I/R injury by activating Nrf2 pathway.

\footnotetext{
${ }^{1}$ Department of Liver Surgery, Renji Hospital, School of Medicine, Shanghai Jiao Tong University, Shanghai, China; ${ }^{2}$ School of Biomedical Engineering and Med-X Research Institute, Shanghai Jiao Tong University, Shanghai, China; ${ }^{3}$ Animal Laboratory, Renji Hospital, School of Medicine, Shanghai Jiao Tong University, Shanghai, China and ${ }^{4}$ State Key Laboratory of Cell Biology, CAS Center for Excellence in Molecular Cell Science, Innovation Center for Cell Signaling Network, Institute of Biochemistry and Cell Biology, Shanghai Institutes for Biological Sciences, Chinese Academy of Sciences, Shanghai, China

*Corresponding author: X Wang or Q Xia or X Kong, Department of Liver Surgery, Renji Hospital, School of Medicine, Shanghai Jiao Tong University, 160 Pujian Road, Shanghai 200127, China. Tel: +86 21 68383283; Fax: +86 21587372 32; E-mail: doctorwangxin@163.com or xiaqiang@ medmail.com.cn or xiaoni-kong@126.com

${ }^{5}$ These authors contributed equally to this work.

Received 12.4.17; revised 03.7.17; accepted 12.7.17; Edited by C Munoz-Pinedo
} 
Autophagy is a cytoprotective process involving in degradation of long-lived cytoplasmic proteins, surplus or dysfunctional organelles through a lysosome-dependent machinery to maintain cellular homeostasis. ${ }^{18}$ Numerous studies have shown that autophagy has crucial roles in liver pathology such as metabolic diseases, infectious diseases and hepatocellular carcinoma. ${ }^{19}$ So far the role of autophagy in the pathogenesis of I/R injury is controversial. ${ }^{20,21}$ Our previous study indicates that induction of autophagy protects liver from I/R injury. ${ }^{22}$ Here, we further demonstrate that CDDO-Im protects hepatic I/R injury by activating the Nrf2-HO-1-autophagy axis.

In this study, we investigated the role of CDDO-Im in hepatic I/R injury and demonstrated that CDDO-Im could ameliorate

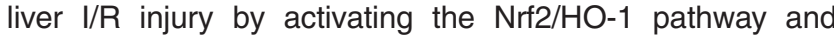
induction of autophagy, which is responsible for clearance of damaged mitochondria and reduced production of ROS and proinflammatory cytokines. Given that mitochondrial dysfunction is a major early detrimental event contributing to induction of high ROS and inflammatory response in I/R injury, CDDOIm preconditioning may be a potent therapeutic approach to target this early stage of $\mathrm{I} / \mathrm{R}$ injury process.

\section{Results}

CDDO-Im protects liver from I/R injury. CDDO-Im is a well-known activator of Nrf2. ${ }^{13,17}$ To assess whether CDDOIm treatment has a protective role on liver l/R injury, we started to determine the proper treatment dose and time of CDDO-Im in wild-type (WT) mice by measuring the transcriptional induction of Nrf2 target genes such as Gclc, Gclm, Gstm1, Ho1 and Nqo1 in liver tissues. As shown in Figures $1 \mathrm{a}$ and $\mathrm{b}, 2 \mathrm{mg} / \mathrm{kg}$ of CDDO-Im treatment for $3 \mathrm{~h}$ could significantly increase the hepatic expression of those genes. In our liver I/R model, mice pretreated with CDDO-Im or vehicle were subjected to $90-\mathrm{mi}$ hepatic $70 \%$ ischemia, followed by reperfusion and the liver I/R injury was assessed by biochemical measurements and histopathology analyses. As shown in Figure 1c, compared with mice pretreated with vehicle whose hepatic necrotic areas were markedly increased $6 \mathrm{~h}$ and $12 \mathrm{~h}$ post reperfusion, CDDO-Im pretreated mice showed great resistance to l/R-induced liver injury. In line with the decreased liver injury, the liver sections of CDDO-Im pretreated mice were graded with significantly lower Suzuki scores than those in the vehicle group (Figure 1d). Consistent with these histological alterations, CDDO-Im pretreatment also markedly decreased serum aspartate aminotransferase (AST) and alanine transaminase (ALT) levels (Figure 1e). These results clearly demonstrated that CDDO-Im pretreatment attenuates the detrimental effects of hepatic I/R injury.

CDDO-Im alleviates inflammatory responses during hepatic I/R injury. Given that the activation of the inflammatory response plays an important role in exacerbating hepatic I/R injury, we started to assess the accumulation of hepatic inflammatory cells by staining myeloperoxidase (MPO) (a neutrophil marker) and F4/80 (a macrophage marker) after I/R injury. As shown in Figures 2a-d, CDDO-Im treatment resulted in significantly decreased hepatic infiltration of both neutrophils and macrophages after reperfusion compared with the vehicle control group. In response to the reduced hepatic infiltration of innate immune cells, significantly less induction of proinflammatory cytokines such as IL- $1 \beta$, IL- 6 , TNF- $\alpha$ and CXCL-10 was detected in the CDDO-Im group compared with the vehicle group $6 \mathrm{~h}$ after I/R treatment (Figures $2 e$ and $f$ ). These findings clearly demonstrated that CDDO-Im pretreatment alleviates the inflammatory responses following hepatic I/R treatment.

CDDO-Im reduces hepatocyte apoptosis in hepatic I/R injury. As $\mathrm{l} / \mathrm{R}$ injury is associated with liver apoptosis, which is mainly mediated by TNF-a, ${ }^{23}$ we then assessed the hepatic apoptosis levels in both CDDO-Im-treated and untreated groups by terminal deoxynucleotidyl transferase dUTP nick end labeling (TUNEL) assays. Although I/R induced marked DNA damages in both groups, CDDO-Im treated mice displayed significantly reduced TUNEL signals in the liver sections compared with control mice (Figures $3 a$ and b). In line with the TUNEL assay results, immunohistochemistry (IHC) staining of cleaved caspase-3 also showed decreased signals in the CDDO-Im treated group compared with the untreated one (Figures $3 c$ and $d$ ). This reduced apoptosis in CDDO-Im treated mice is at least partially due to increased expression of anti-apoptotic proteins, such as $\mathrm{Bcl} 2$ and $\mathrm{Bcl}-\mathrm{xl}$ because western blot showed an increase of those two genes in the CDDO-Im treated group compared with the control group (Figure $3 e$ ). In addition, the caspase-3 activities were markedly decreased in the CDDO-Im group compared with the control group (Figure $3 f)$. Given that previous studies have reported the positive regulation of Nrf2 on the expression of $\mathrm{Bcl} 2$ and $\mathrm{Bcl}-\mathrm{xl}^{24}$ it is not surprising to detect elevated $\mathrm{Bcl} 2$ and $\mathrm{Bcl}-\mathrm{xl}$ levels in the CDDO-Im treated group. To further confirm the protective effect of CDDO-Im on hepatocyte apoptosis during I/R injury, we isolated primary hepatocytes from WT mice and pretreated them with or without CDDO-Im in vitro followed with hypoxia/reoxygenation $(H / R)$ to mimic in vivo $l / R$ injuries. $H / R$-induced cell damage was determined by Lactate dehydrogenase (LDH) cytotoxicity assays. In line with our in vivo findings, compared with the control group, CDDO-Im pretreatment significantly preserved hepatocytes in $\mathrm{H} / \mathrm{R}$ treatment as demonstrated by reduced $\mathrm{LDH}$ release (Figure $3 \mathrm{~g}$ ). This protection might be due to diminished apoptosis levels, because reduced cleaved caspase-3 and increased $\mathrm{Bcl} 2$ and $\mathrm{Bcl}-\mathrm{xl}$ levels were detected in the hepatocytes treated with CDDO-Im after $\mathrm{H}$ / $\mathrm{R}$ (Figure 3h). Therefore, these results clearly demonstrated that CDDO-Im protects hepatocytes against I/R induced apoptosis.

CDDO-Im enhances liver autophagy during hepatic I/R injury. Autophagy has emerged recently as an important safeguard against I/R injury by clean-up damaged mitochondria and in turn inhibiting excessive ROS production. ${ }^{25}$ We then sought to investigate whether CDDO-Im affects autophagy during hepatic I/R injury. As shown in Figure $4 a$, levels of LC3b-II, a standard indicator of autophagy activity, were greater in the CDDO-Im pretreated mice than that in the control mice $6 \mathrm{~h}$ after I/R injury. Moreover, compared with the controls, CDDO-Im pretreatment resulted in a marked 

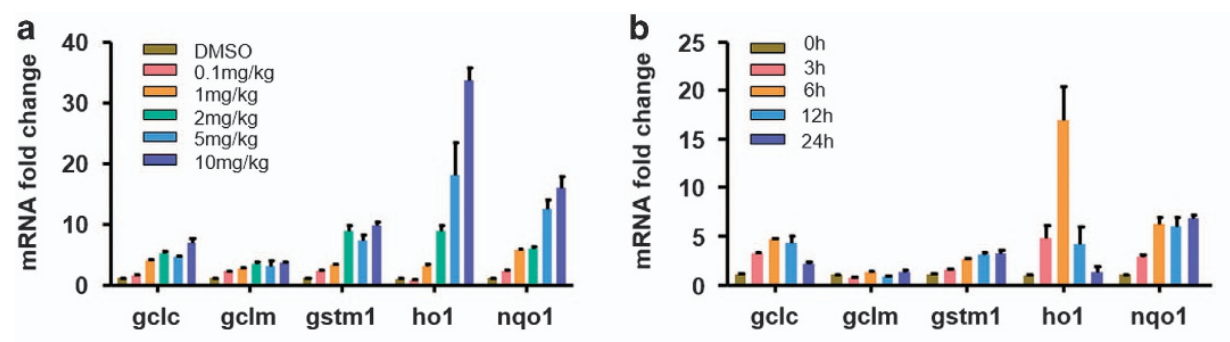

c
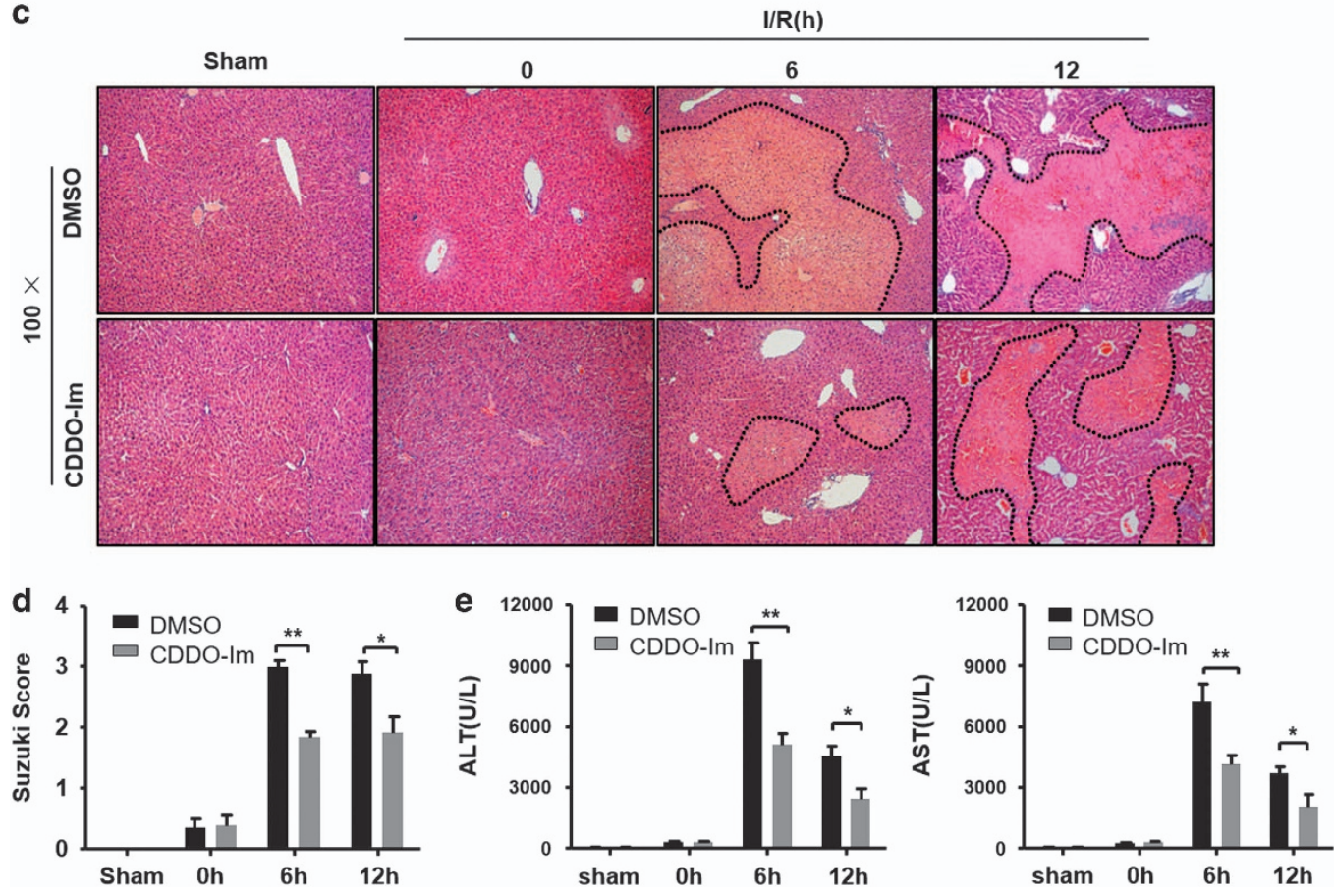

Figure 1 CDDO-Im pretreatment attenuates I/R injury. (a,b) Time-course $(0-24 \mathrm{~h})$ and dose-response $(0-10 \mathrm{mg} / \mathrm{kg})$ of CDDO-Im pretreatment and Nrf2 target genes mRNA levels ( $n=3$ per group). (c) Representative hematoxylin and eosin (HE)-stained sections (original magnification, $\times 100$ ) and relevant average Suzuki score (d) at $0,6,12 \mathrm{~h}$ post reperfusion or sham controls. (mean \pm S.E.M., $n=4-6$ per group). (e) Serum ALT and AST levels at $0,6,12 \mathrm{~h}$ post reperfusion; mean \pm S.E.M., $n=4-6$ per group. ${ }^{\star \star} P<0.01$, ${ }^{*} P<0.05$

decrease in the levels of SQSTM1/p62, a classic macroautophagy substrate (Figure 4a). Autophagy is a highly dynamic and multi-step process. Therefore, LC3b-II levels at certain time point are insufficient to represent autophagy flux. We then employed Chloroquine (CQ) to block autophagy flux in mice with or without CDDO-Im pretreatment. Although CQ treatment significantly increased the LC3B-II levels in both groups, CDDO-Im pretreated mice showed greater LC3B-II elevation as compared with controls (Figures $4 \mathrm{~b}$ and $\mathrm{C}$ ). Correspondingly, analysis of autophagy using transmission electron microscope (TEM) showed increased numbers of autophagosomes in the liver of CDDO-Im pretreatment mice compared with the control mice $6 \mathrm{~h}$ after I/R injury (Figures $4 \mathrm{~d}$ and e). Meanwhile, we similarly investigated the effect of CDDO-Im on autophagy induction in primary hepatocytes in vitro after $\mathrm{H} / \mathrm{R}$ injury. In line with our in vivo findings, compared with DMSO control, CDDO-Im treatment significantly elevated autophagy activity during $H / R$ injury as evidenced by substantially increased LC3B-II levels and decreased SQSTM1/p62 levels (Figure 4f). CQ-mediated autophagy flux blocking assays also showed enhanced autophagy flux in CDDO-Im treated hepatocytes compared with DMSO controls (Figures $4 \mathrm{~g}$ and $\mathrm{h}$ ). Meanwhile, accumulation of autophagic vacuoles was detected by CYTO-ID autopahy detection kit. As shown in Figures 4i and $\mathrm{j}$, autophagic vesicles in CDDO-Im pretreatment hepatocytes were more abundant than that in control hepatocytes after $H / R$ injury. These results suggested that CDDO-Im pretreatment enhances autophagy during hepatic I/R injury.

CDDO-Im protects against mitochondrial dysfunction and excessive ROS induction during liver $\mathrm{I} / \mathrm{R}$ injury. Given the important role of autophagy in removal of damaged and ROS overproduction mitochondria, we accessed the presence of damaged mitochondria in both groups by detecting circulating mitochondrial DNA (mtDNA) in serum. In line with the differential autophagy activities in these two groups, the amount of the circulating mtDNA was markedly lower in CDDO-Im pretreated mice compared with control mice (Figure 5a). In response to the less presence of damaged mitochondria, reduced ROS levels were detected in CDDO-Im pretreated mice as demonstrated by decreased 

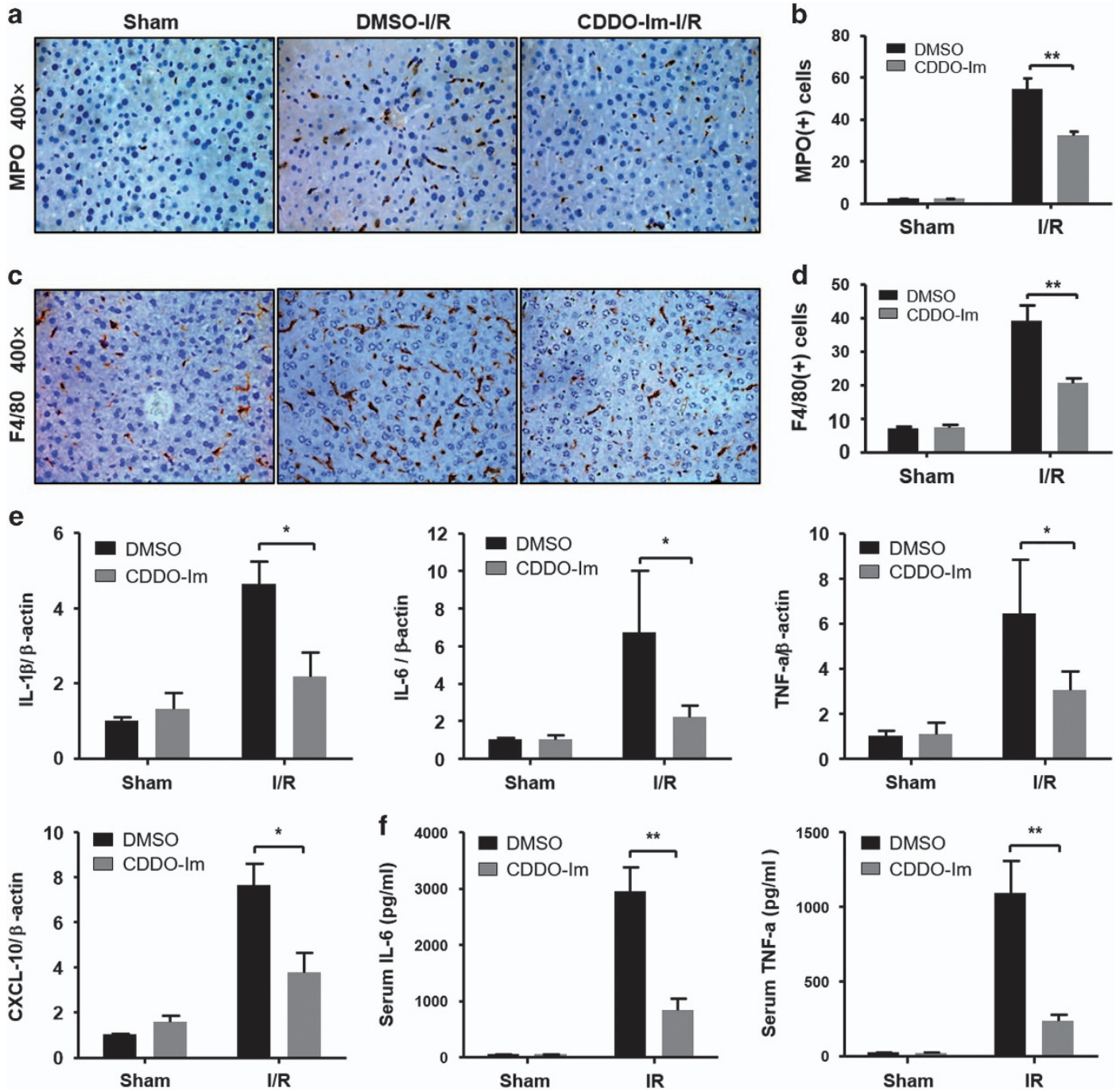

Figure 2 CDDO-Im depresses neutrophil infiltration and reduces cytokine release. (a) Representative sections from MPO staining and F4/80 staining (c) at $6 \mathrm{~h}$ after reperfusion (original magnification, $\times 400$ ), the numbers of MPO (b) and F4/80 (d) positive cells that infiltrated the livers were determined. (e) mRNA levels of cytokines and chemokines were determined by quantitative real-time PCR, and (f) Serum cytokine levels measured by ELISA. All data shown as the mean $\pm S$.E.M., ${ }^{* *} P<0.01,{ }^{*} P<0.05$

malonaldehyde (MDA) levels (Figure 5b). To further confirm the protective role of CDDO-Im in mitochondrial dysfunction and ROS overproduction, primary hepatocytes from WT mice were treated with $H / R$ injury in vitro. Mitochondrial injury and mitochondrial ROS levels were accessed via mitochondrial membrane potential $(\Delta \psi \mathrm{m})$ (using JC-1 fluorescent dye) and mitochondrial ROS (using mitoSOX Red dye), respectively. As shown in Figures $5 \mathrm{c}$ and d, compared with the DMSO control, CDDO-Im treatment displayed protection on $\Delta \psi \mathrm{m}$ as indicated by decreased ratio of green to red fluorescence intensity. In addition, hepatocytes with CDDOIm treatment showed less mitochondrial ROS production as evidenced by reduced MitoSOX Red fluorescent signals (Figures $5 e$ and f).

Autophagy accounts for CDDO-Im-mediated protection in liver $\mathbf{I} / \mathbf{R}$ injury. To test whether the cytoprotection of
CDDO-Im was mediated by induction of autophagy, we adopted a pharmacological autophagy inhibitor 3-methyladenine (3-MA) to block autophagy in both groups during $1 / R$ injury. As shown in Figure $6 a$, blocking of autophagy remarkably increased the serum ALT/AST levels in the CDDO-Im pretreated group but not in the control group. Consistently, hematoxylin and eosin (HE) staining showed greatly increased necrotic areas in the liver of CDDO-Im pretreated mice owing to autophagy blockage (Figures $6 \mathrm{~b}$ and $\mathrm{c}$ ). In addition, 3-MA treatment showed a significant autophagy inhibition in the CDDO-Im group as demonstrated by decreased cellular autophagosomes (Figures $6 \mathrm{~d}$ and e). Correspondingly, autophagy inhibition resulted in hepatocytes of CDDO-Im group more sensitive to $\mathrm{H} / \mathrm{R}$ induced cell injury as indicated by increased LDH levels (Figure 6f). These findings clearly indicated that CDDO-Immediated hepatic protection against $\mathrm{I} / \mathrm{R}$ injury mainly depend on autophagy induction. 
a

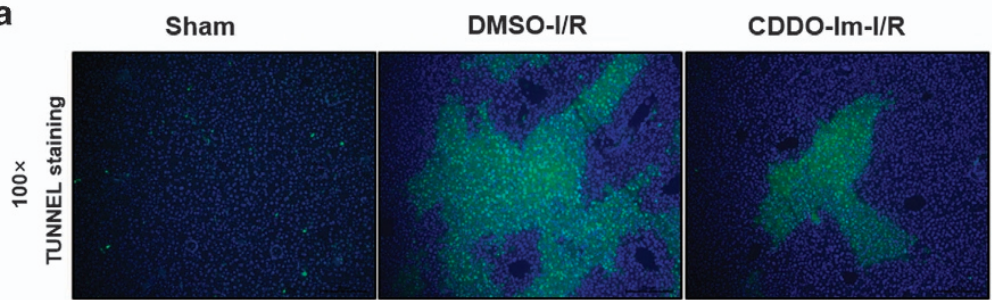

C

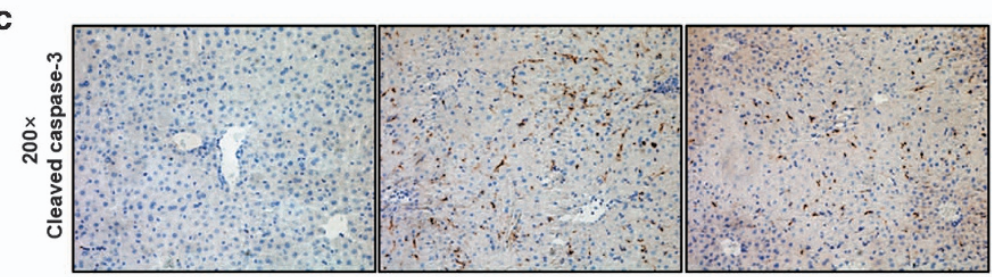

b

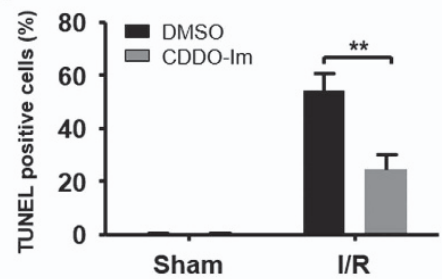

d

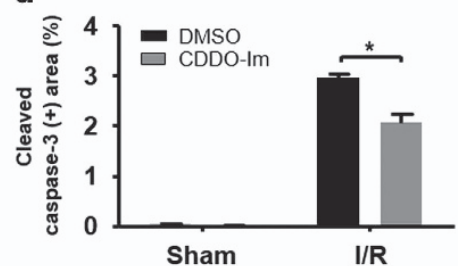

e
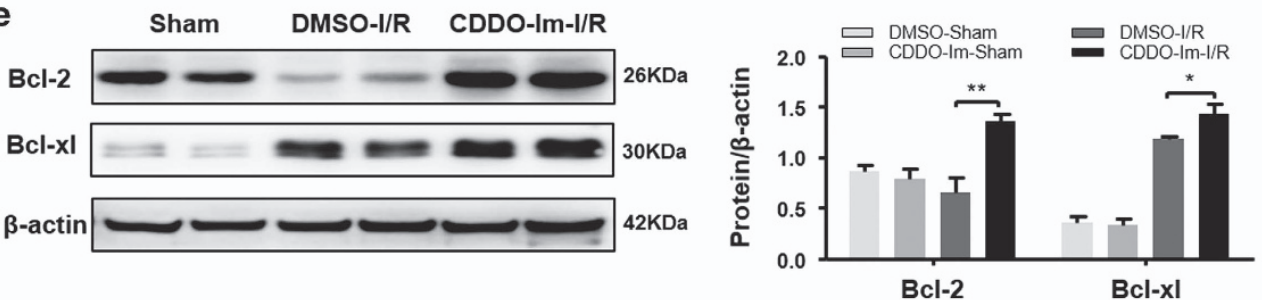

f
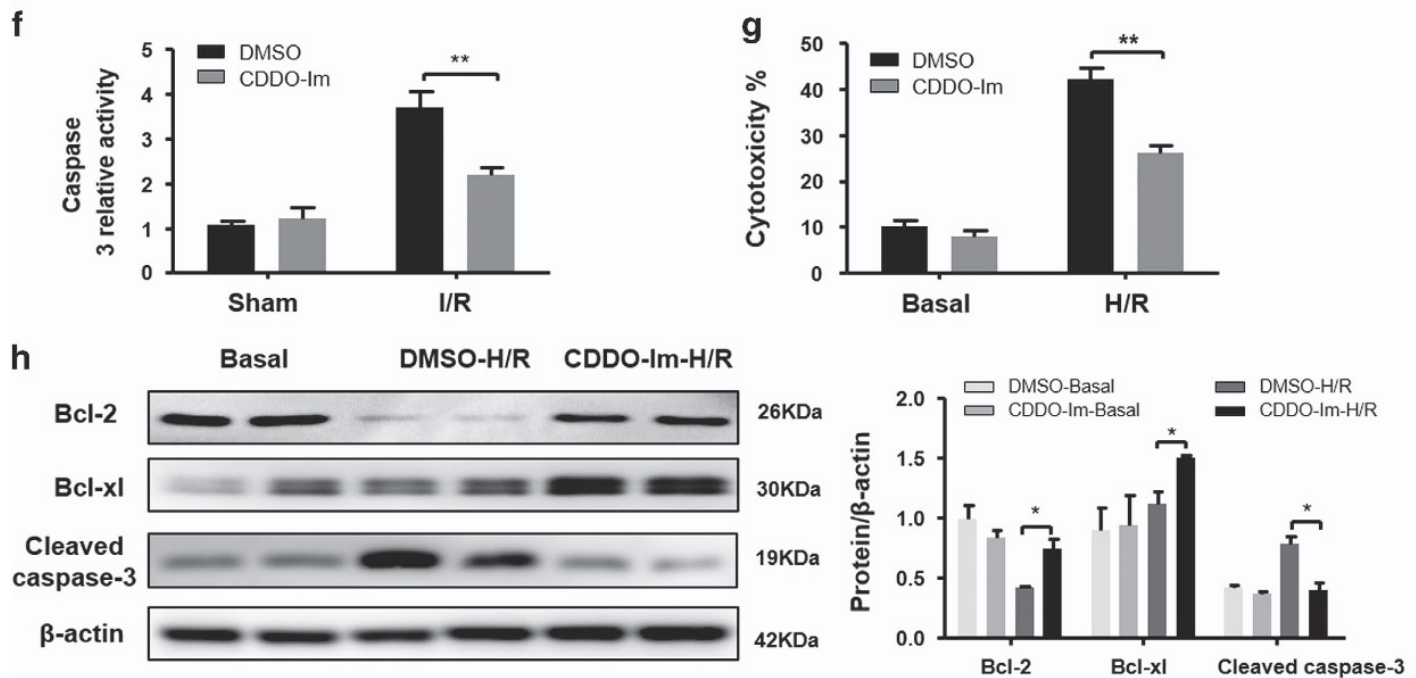

Figure $3 \mathrm{CDDO}-\mathrm{Im}$ pretreatment alleviate apoptosis in hepatic I/R injury both in vitro and vivo. (a,b) Representative sections of TUNEL staining at $6 \mathrm{~h}$ after reperfusion. TUNEL-positive hepatocytes were expressed as a percentage of the total hepatocytes. (c,d) Representative sections from cleaved caspase-3 staining (original magnification, $\times 200$ ), the cleaved caspase-3-positive area that infiltrated the livers were determined. (e) Western blot analysis of Bcl2 and Bcl-xl in the livers of DMSO and CDDO-Im pretreatment mice at $6 \mathrm{~h}$ after I/R or a sham operation. $\beta$-actin served as a loading control. (f) Caspase-3 activity was evaluated from a total lysate of I/R lobes or sham mice from CDDO-Im or DMSO pretreated group. ( $n=3-4$ per group, mean \pm S.E.M., $\left.{ }^{* \star} P<0.01,{ }^{*} P<0.05\right)$. (g) Cell death of isolated primary hepatocytes after $\mathrm{H} / \mathrm{R}$ injury were measured by LDH assays. Average cytotoxicity (\% cell death) in different groups were plotted. Three to five replicates per experiment group. (h) Bcl2, Bcl-xl and cleaved caspase-3 protein expression were analyzed by western blotting in isolated hepatocytes from WT with or without CDDO-Im pretreatment subjected to $\mathrm{H} / \mathrm{R}$ for $4 \mathrm{~h}$. $(n=3-4$ per group, mean \pm S.E.M., $\left.{ }^{\star \star} P<0.01,{ }^{*} P<0.05\right)$

CDDO-Im-induced autophagy is dependent on Nrf2/HO-1 Pathway. As CDDO-Im is a well known potent pharmacological Nrf2 activator by promoting nuclear translocation of $\mathrm{Nrf}^{26}$ and $\mathrm{HO}-1$, a target gene of Nrf2, has been reported to reduce liver injury by induction of autophagy via an unknown mechanism, ${ }^{27,28}$ we further investigated whether CDDO-Immediated autophagy is through the $\mathrm{Nrf} / \mathrm{HO}-1$ pathway. I/R injury-mediated Nrf2 activation was confirmed by western blot as indicated by an increase in nuclear Nrf2 levels and a decreased in cytoplasmic Nrf2 levels (Figure 7a). Interestingly, although the nuclear Nrf2 levels seemed comparable in both DMSO control and CDDO-Im groups, CDDO-Im pretreatment substantially enhanced $\mathrm{HO}-1$ expression (Figure 7a). This CDDO-Im pretreatment associated 
elevation was further confirmed by $\mathrm{IHC}$ staining of $\mathrm{HO}-1$ in the liver sections after I/R injury (Supplementary Figure S1A). We next employed Nrf2 knockout (KO) mice to further identify whether CDDO-Im-mediated protection and autophagy are the Nrf2-dependent. Nrf2 depletion abrogated CDDO-Immediated protection against liver I/R injury as demonstrated

a
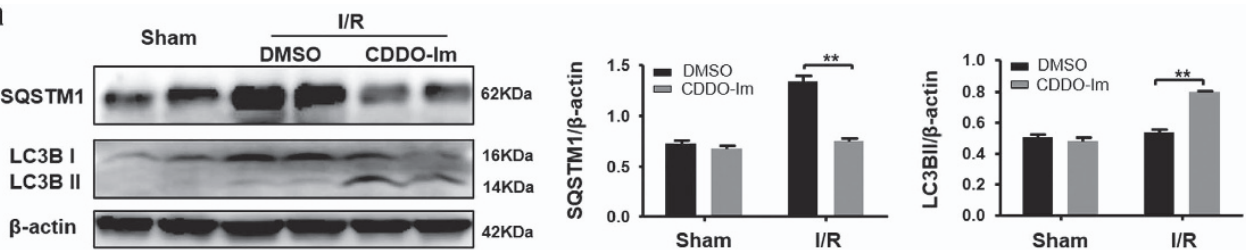

b
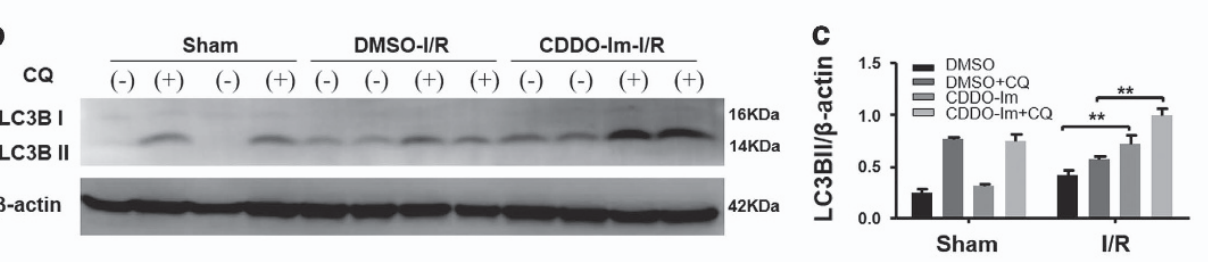

d

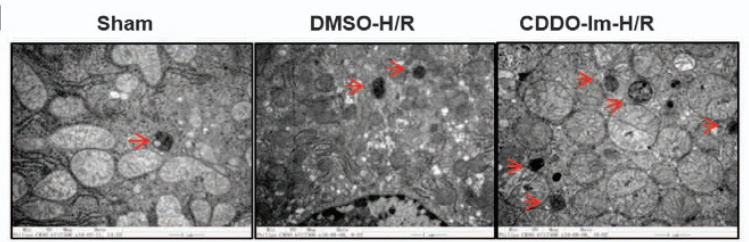

e

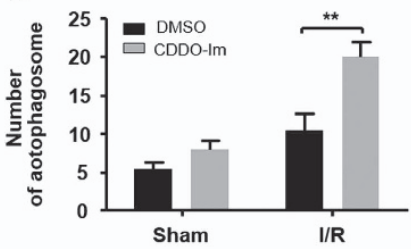

f
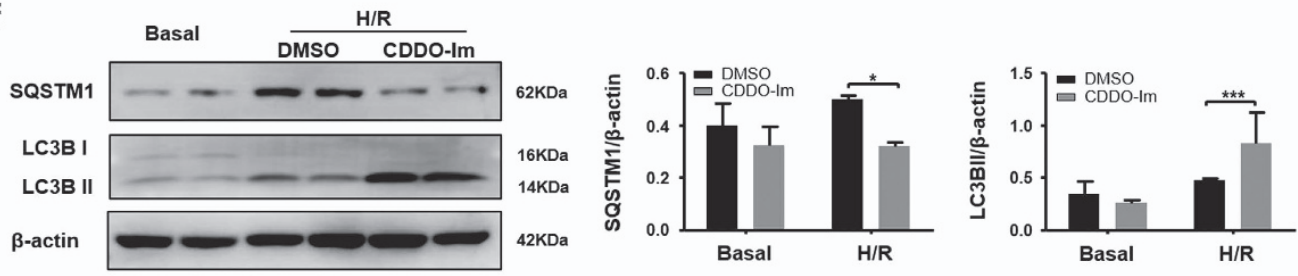

g
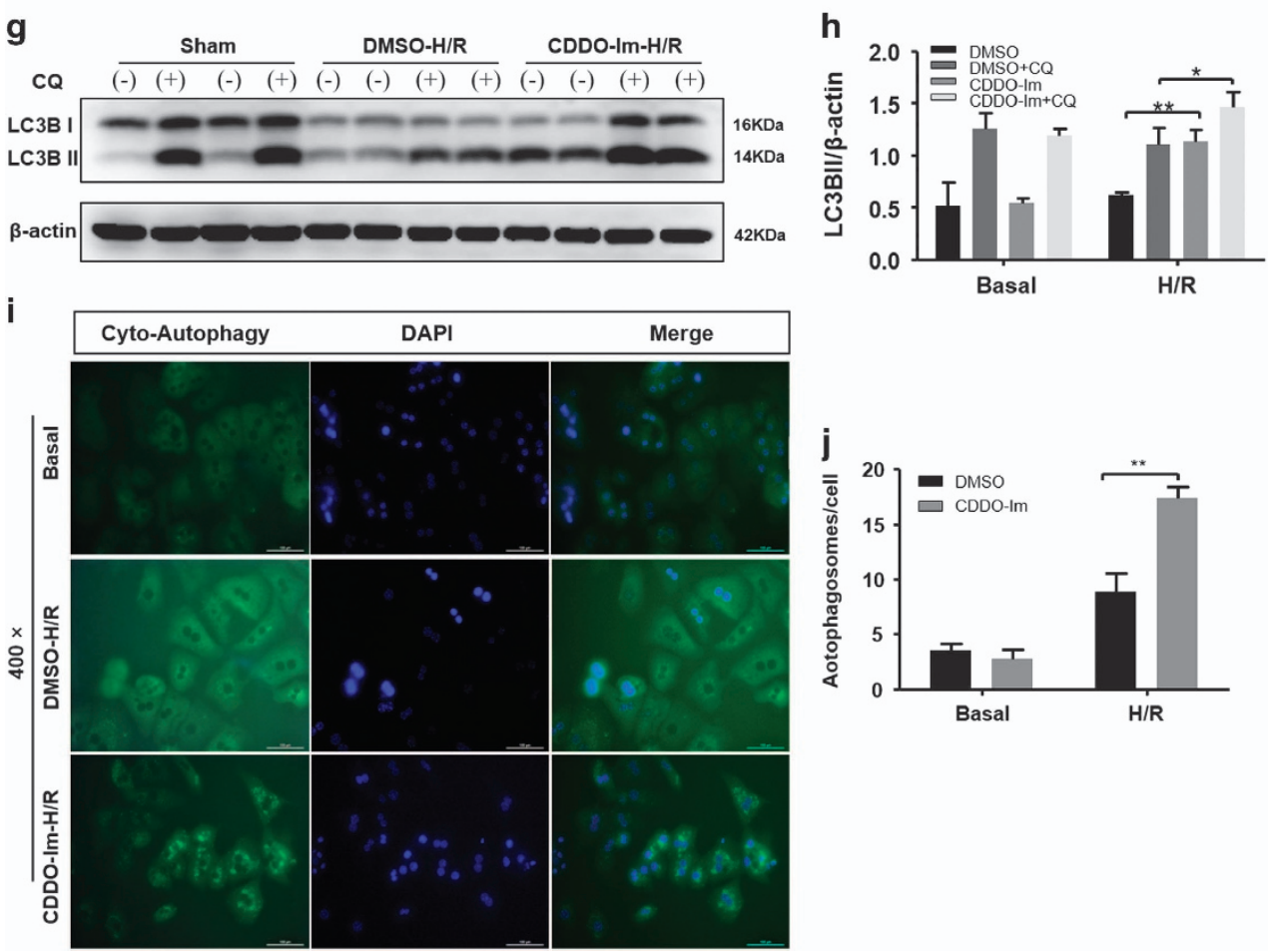
a

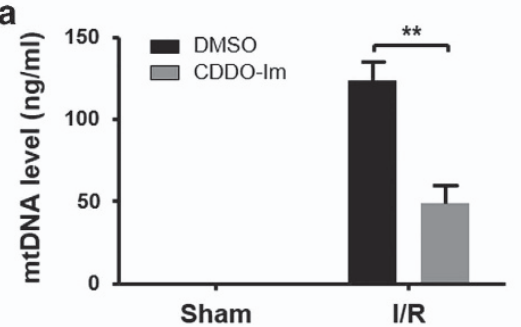

C

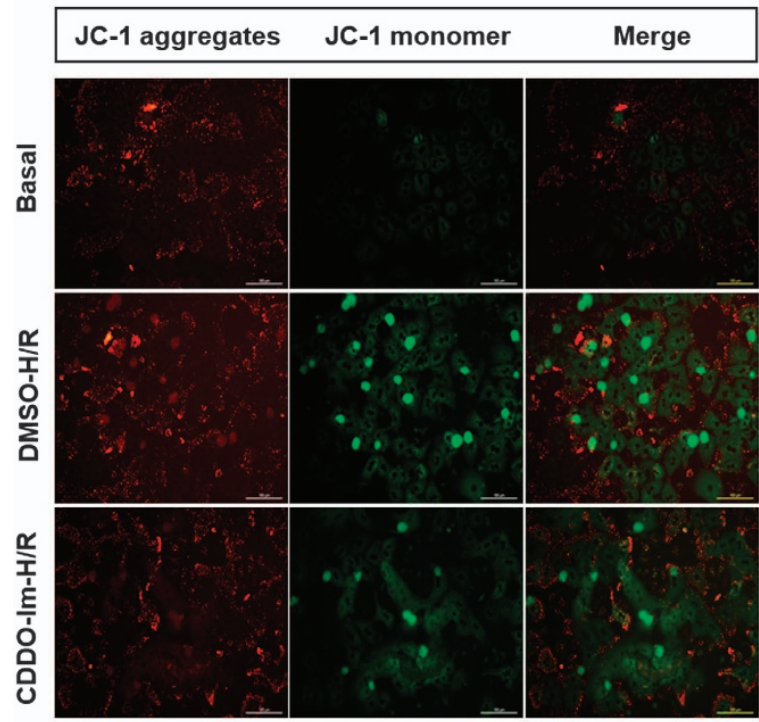

d

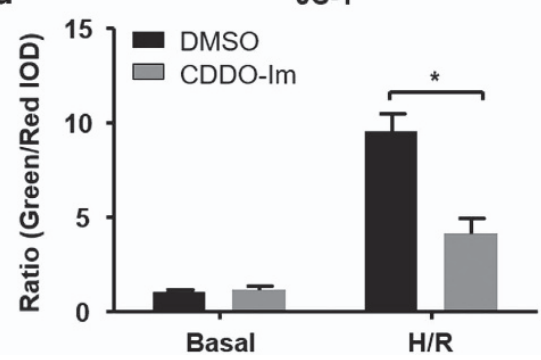

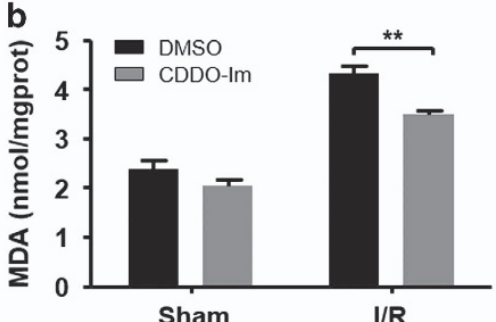

e

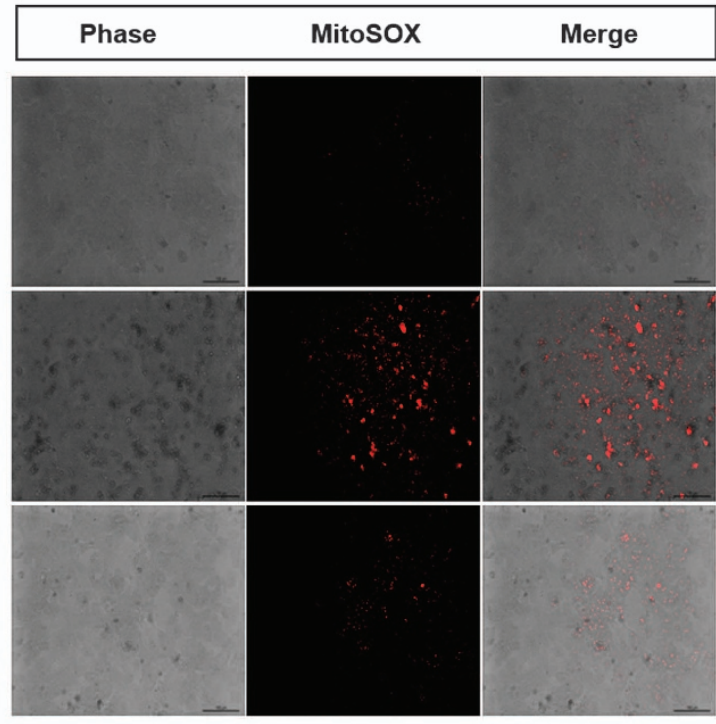

f

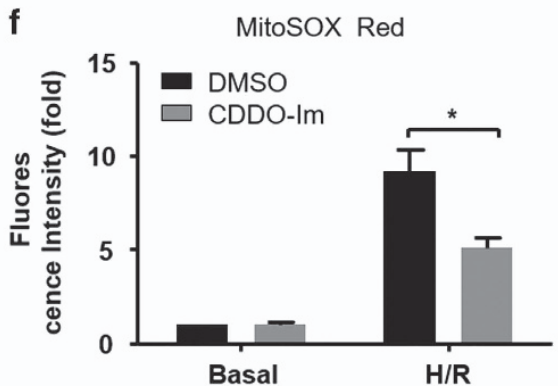

Figure 5 CDDO-Im pretreatment inhibit oxidative stress and protect against mitochondrial dysfunction during hepatic l/R injury. (a) Serum levels of mtDNA in mice at $6 \mathrm{~h}$ after reperfusion. (b) Effects of the CDDO-Im and DMSO on the levels of MDA in mice livers at $6 \mathrm{~h}$ after reperfusion. (c, d) Representative pictures of mitochondrial membrane potential of primary hepatocytes isolated from WT, which subjected to H/R injury in the CDDO-Im and DMSO pretreatment group (original magnification, $\times 400$ ). The ratio of green to red fluorescence intensity were determined. (e,f) Representative pictures of mitochondrial ROS accumulation of primary hepatocytes after $\mathrm{H} / \mathrm{R}$ injury in both groups (original magnification, $\times 200)$. The mitochondrial ROS was expressed by relative red area of the total picture. Values expressed as mean \pm S.E.M. $(n=4-6)$. ${ }^{*} P<0.05$ and ${ }^{* \star} P<0.01$

Figure 4 CDDO-Im pretreatment enhances liver autophagy induction in hepatic I/R injury. (a) Western blot analysis of LC3B and SQSTM1 protein expression in the CDDO-Im and DMSO pretreatment group at $6 \mathrm{~h}$ after reperfusion. $\beta$-actin served as a loading control. Coresponding densitometric analysis of LC3B-II and SQSTM1 expression. (b,c) Western blot analysis of LC3B protein expression in the presence and absence of chloroquine $(60 \mathrm{mg} / \mathrm{kg})$ and densitometric analysis of LC3B-II expression. $\beta$-actin was used as the loading control. (d,e) Representative transmission electron micrographs showing autophagosomes in the ischemic lobes at $6 \mathrm{~h}$ of reperfusion. Autophagosomes are indicated by arrows. Scale bars $1 \mu \mathrm{m}$. The numbers of autophagosomes were determined. The data are shown as the mean \pm S.E.M. $n=4-6$ per group. (f) Western blot analysis of LC3B and SQSTM1 protein expression in the CDDO-Im and DMSO pretreatment primary hepatocytes isolated from WT, which subjected to H/R injury. $\beta$-actin served as a loading control. Coresponding densitometric analysis of LC3B-II and SQTM1 expression. (g,h) Western blot analysis of LC3B protein expression in the presence and absence of chloroquine $(50 \mathrm{mM})$ and densitometric analysis of LC3B-II expression. $\beta$-actin was used as the loading control. (i,j) Representative fluorescence micrographs display autophagosomes in hepatocytes with CDDO-Im and DMSO pretreatment (original magnification, $\times 400$ ). The numbers of autophagosomes were determined. The data are shown as the mean \pm S.E.M. $n=4-6$ per group 
a

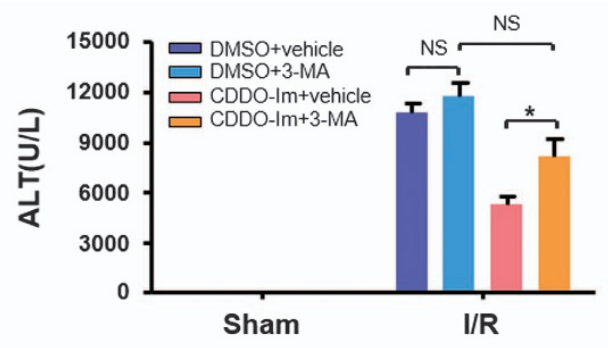

b
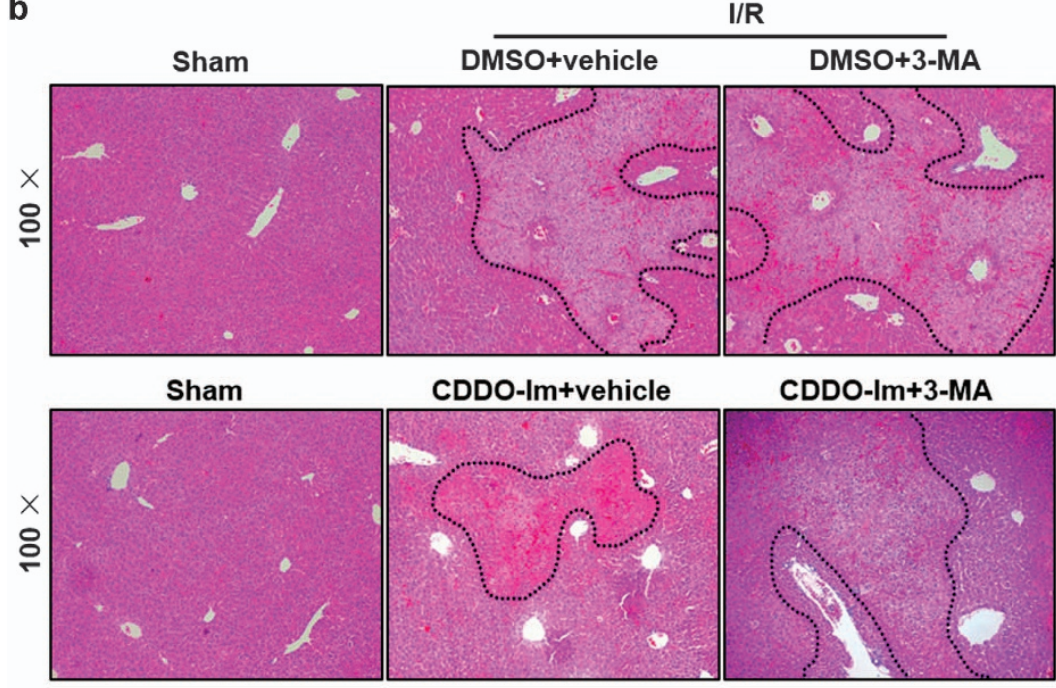

d

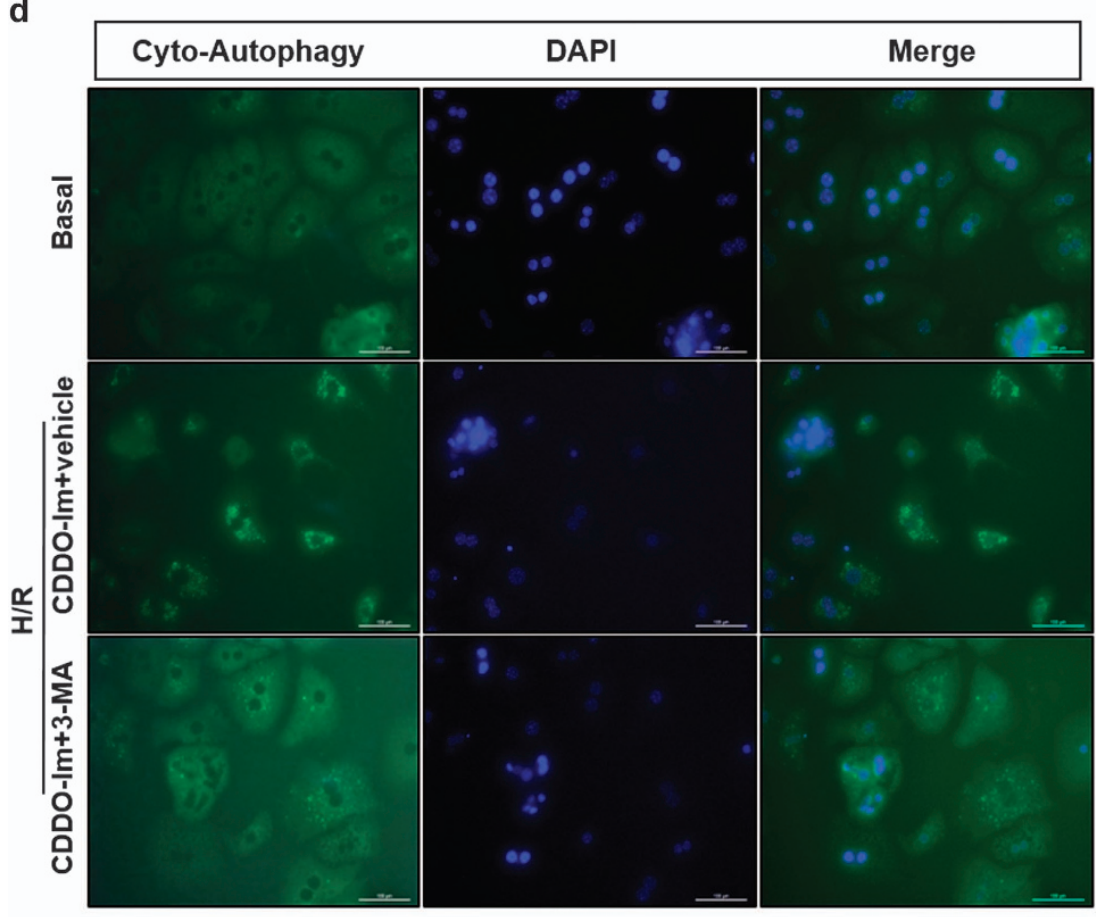

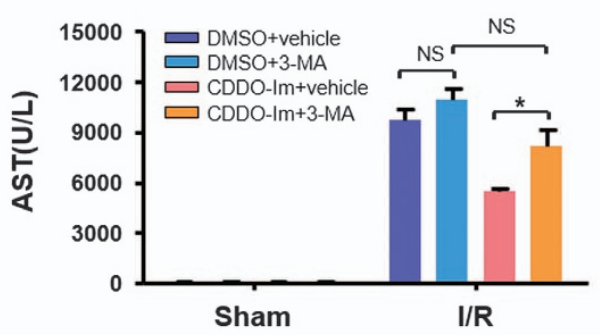

C

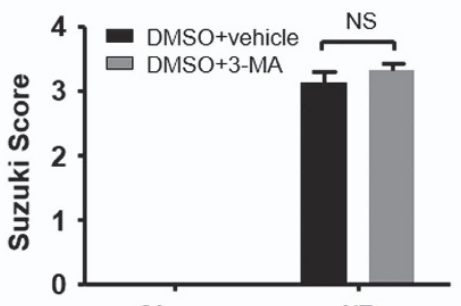

Sham
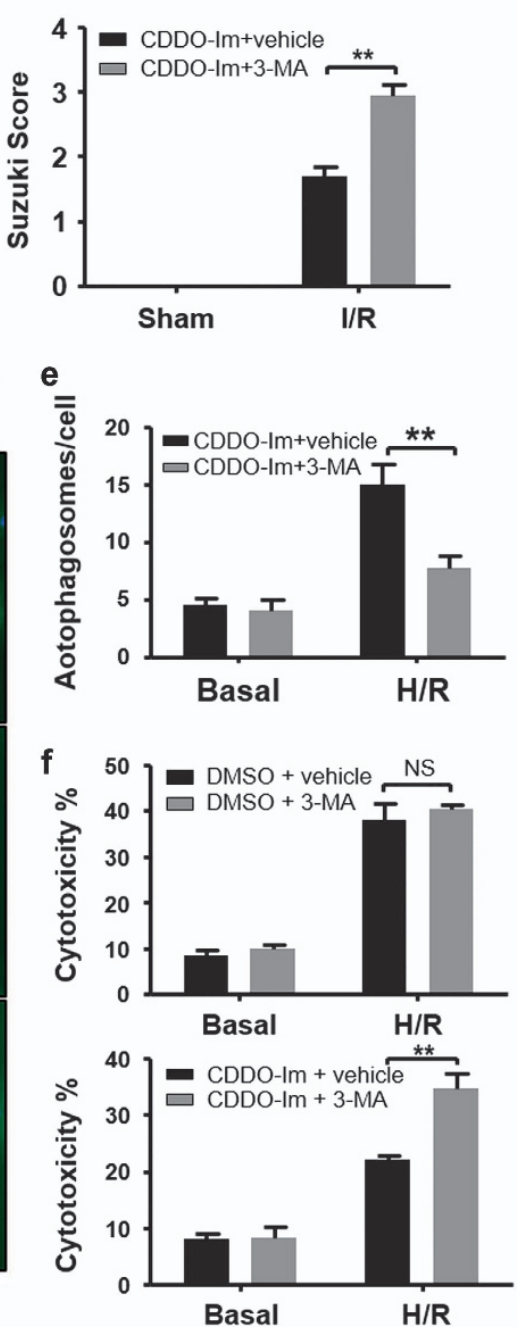

Figure 6 CDDO-Im pretreatment protected against liver I/R injury by means of autophagy induction. (a-c) Mice were pretreated with 3-Methyladenine (30 $\mathrm{mg} / \mathrm{kg}$, IP) $2 \mathrm{~h}$ after CDDO-Im (2 mg/kg, IP) or DMSO and killed at $6 \mathrm{~h}$ after reperfusion. (a) Serum ALT and AST levels. (b) Representative hematoxylin and eosin (HE)-stained sections (original magnification, $\times 100)$ and (c) relevant average Suzuki score. (d, e) Representative fluorescence micrographs display autophagosomes in primary hepatocytes with 3-MA in the presence or absence of CDDO-Im (original magnification, $\times 400$ ). The numbers of autophagosomes were determined. (f) Quantification of average cytotoxicity $(\%$ cell death) in different groups were plotted. ( $n=3-4$ per group, mean \pm S.E.M., $\left.{ }^{* \star} P<0.01,{ }^{\star} P<0.05\right)$ 
by increased serum ALT/AST levels and necrotic areas (Figures $7 \mathrm{~b}$ and $\mathrm{c}$ ). In addition, CDDO-Im-mediated HO-1 induction and autophagy enhancement were dramatically decreased in the Nrf2 KO mice (Figure 7d). To further confirm that COOD-Im-mediated cytoprotection and autophagy are Nrf2-dependent, we performed Nrf2 RNAi in primary hepatocytes followed with H/R injury. As shown in Supplementary Figure S2A, Nrf2 RNAi could efficiently knockdown Nrf2 levels in both the cell nucleus and cytoplasm subsets. In accordance with the in vivo results, expression of $\mathrm{HO}-1$ and autophagy activity were remarkably decreased, whereas the $\mathrm{H} / \mathrm{R}$ induced cytotoxicity was significantly increased in Nrf2 knockdown hepatocytes after CDDO-Im treatment (Figures 7e and f). Therefore, these results clearly demonstrate that CDDO-Im-mediated autophagy and protection against liver I/R injury are dependent on Nrf2.

CDDO-Im-mediated autophagy depends on induction of HO-1. To determine whether HO-1 is required for CDDO-Immediated autophagy and hepatic protection against I/R injury, we employed a HO-1 inhibitor Tin Protoporphyrin IX dichlorid (SnPP) to treat WT mice followed with I/R injury. Administration of SnPP abolished CDDO-Im-mediated hepatic protection as demonstrate by increased serum ALT/AST levels and necrotic areas (Figures $8 \mathrm{a}$ and $\mathrm{b}$ ), indicated that the protective effects of CDDO-Im were dependent on $\mathrm{HO}-1$. In addition, CDDO-Im-mediated autophagy was also remarkably decreased in SnPP treated mice as indicated by declined LC3B-II levels (Figure 8c). To further confirm that COOD-Im-mediated cytoprotection and autophagy are HO-1dependent, we performed $\mathrm{HO}-1 \mathrm{RNAi}$ in primary hepatocytes followed with $H / R$ injury. As shown in Supplementary Figure S2B, HO-1 RNAi efficiently knocked down $\mathrm{HO}-1$ levels. Autophagy activity was significantly decreased in the CDDO-Im group but not the DMSO control as indicated by declined LC3B-II levels (Figure 8d). Meanwhile, the H/Rinduced cytotoxicity was significantly increased in $\mathrm{HO}-1$ knockdown hepatocytes after CDDO-Im treatment as evidenced by increased LDH levels (Figure 8e). In addition, impaired autophagy of CDDO-Im group activity was further confirmed by fluorescent staining of autophagosomes (Figure 8f). These findings strongly indicate that CDDO-Immediated autophagy induction depends on the activation of Nrf2/HO-1.

\section{Discussion}

In present study, we demonstrated that CDDO-Im can improve I/R induced liver injury. Phenotypically, CDDO-Im attenuates hepatocyte necrosis and apoptosis, reduces inflammatory responses and ameliorates mitochondrial dysfunction during liver I/R injury. These protection phenotypes are attributed to CDDO-Im-mediated autophagy enhancement. Mechanistically, by activating Nrf2 and stimulating its downstream target gene $\mathrm{HO}-1$ expression, CDDO-Im enhances $\mathrm{HO}-1$-mediated autophagy, intensifies the clearance of damaged mitochondria and reduces the mtDNA release and ROS overproduction, and in turn dulled the damage-associated molecular patterns (DAMPs) induced inflammatory responses and the following secondary liver injury.
Although necrosis is conventionally recognized as a main detrimental factor during liver $\mathrm{I} / \mathrm{R}$ injury, apoptosis has been implicated recently in the pathogenesis of this process. It is well established that mitochondrial dysfunction is a key trigger to initiate cell death by ATP depletion, uncontrolled ROS surge and final mitochondrial breakdown, which finally lead to cell apoptosis and necrosis. In present study, we demonstrate that CDDO-Im could protect both cell apoptosis and necrosis during I/R injury. CDDO-Im-mediated protection on apoptosis maybe partly due to Nrf2 activation and in turn induction of downstream anti-apoptotic genes such as $\mathrm{Bcl} 2$ and $\mathrm{Bcl}-\mathrm{xl}$. This is consistent with previous studies showing that Nrf2 activation leads to expression of anti-apoptosis genes. In addition, CDDO-Im-mediated autophagy enhancement may also contribute to reduced apoptosis given previous studies that indicate the protection role of autophagy in blocking the onset of apoptosis. Necrosis cells dump tons of DAMPs to further stimulate liver inflammatory responses, which could lead to inflammation-induced secondary liver injury. In present study, we demonstrate that CDDO-Im-mediated autophagy enhancement accounts for its protection on necrosis. By elevating autophagy activity, CDDO-Im augments the clearance of damaged mitochondria, prevents the excessive ROS production and ATP depletion, resulting in reduced cell necrosis.

Autophagy is generally recognized as a cytoprotective mechanism against multiple cellular stresses through its functions including degradation of long-lived cytosolic and damaged proteins, clearing up of damaged mitochondria and modulation of cell death. ${ }^{19,29}$ The protective effects of autophagy during the pathologic process of $\mathrm{I} / \mathrm{R}$ has been identified by several studies. ${ }^{22,30-33}$ In present study, we found enhanced autophagy activity in CDDO-Im group as evidenced by increased expression of LC3B-II and decreased expression of SQSTM1. This enhanced autophagy contributes to CDDOIm-related protection on liver I/R injury because the use of an autophagy inhibitor, 3-MA, almost completely abolished such protection.

A previous study demonstrates that Nrf2 can strengthen autophagy by directly upregulating the expression of Atg3, Atg6 and Atg12 in tumor cells. ${ }^{34}$ However, CDDO-Im-mediated autophagy enhancement is unseemly through the upregulation of those genes. $\mathrm{HO}-1$ as a downstream target gene of Nrf2 has been implicated in protection of hepatic injury during infection/sepsis. ${ }^{35}$ By applying Nrf2 KO mice, we identified the essential role of Nrf2/HO-1 pathway in CDDO-Im-mediated autophagy. Furthermore, by using Nrf2 RNAi and SnPP, an inhibitor of $\mathrm{HO}-1$, we demonstrate that the CDDO-Im-mediated hepatic protection and autophagy enhancement are dependent on $\mathrm{Nrf} / \mathrm{HO}-1$ pathway. Although the mechanism by which $\mathrm{HO}-1$ promotes autophagy activity is not characterized, we hypothesize that carbon monoxide, a product of $\mathrm{HO}-1$ reaction, might be a signaling molecule to stimulate autophagy, because a recent report indicates that carbon monoxide can activate autophagy. ${ }^{36}$

Collectively, our present study indicates that CDDO-Im has a protective role on liver I/R injury by promoting Nrf2/HO-1mediated autophagy activity, which results in increased clearance of damaged mitochondria, reduced production of 
ROS and inflammatory cytokines, and cell death. These findings strongly suggest a therapeutic value of CDDO-Im in liver I/R injury.

\section{Materials and Methods}

Animals. Male WT C57BL/6 mice (8-10 weeks old) were purchased from Shanghai SLAC Co. Ltd (Shanghai, China). Nrf2 KO mice (8-10 weeks old,

a
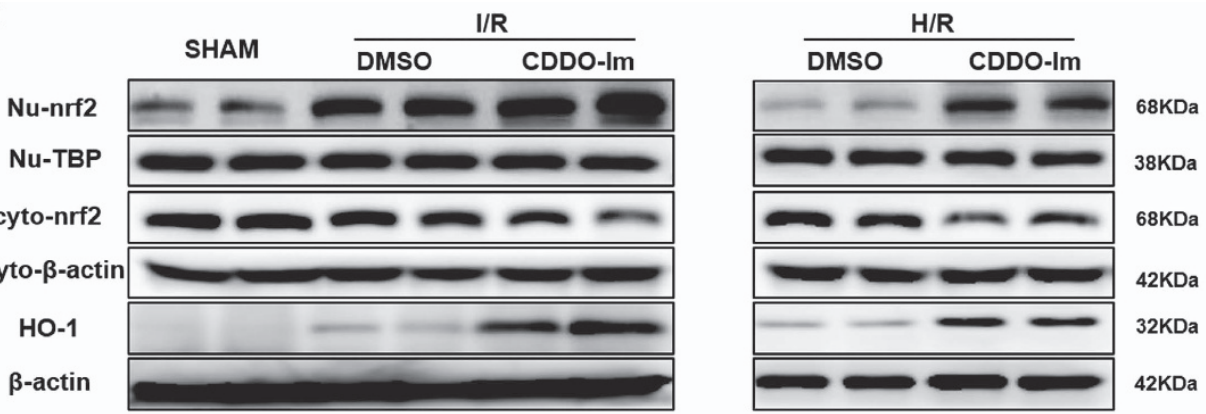

b
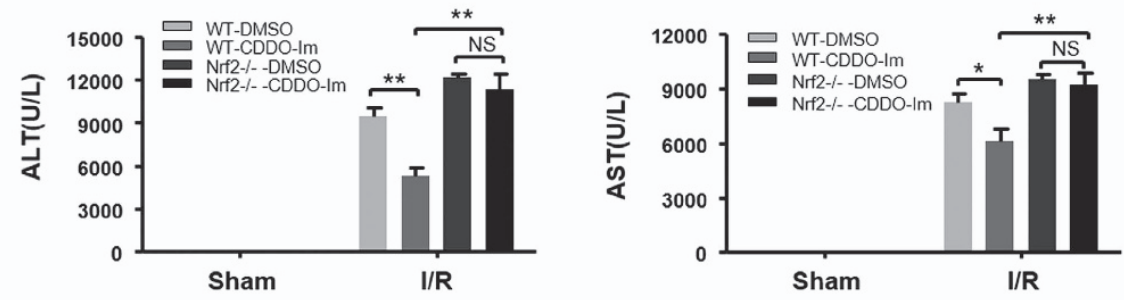

C

I/R

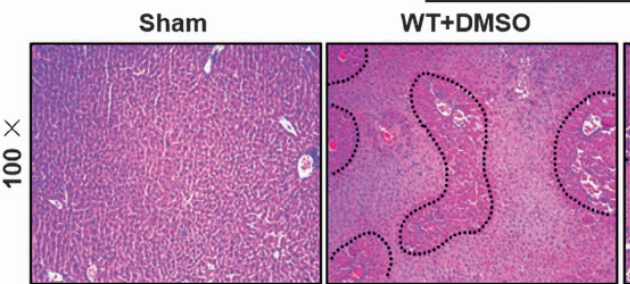

WT+CDDO-Im
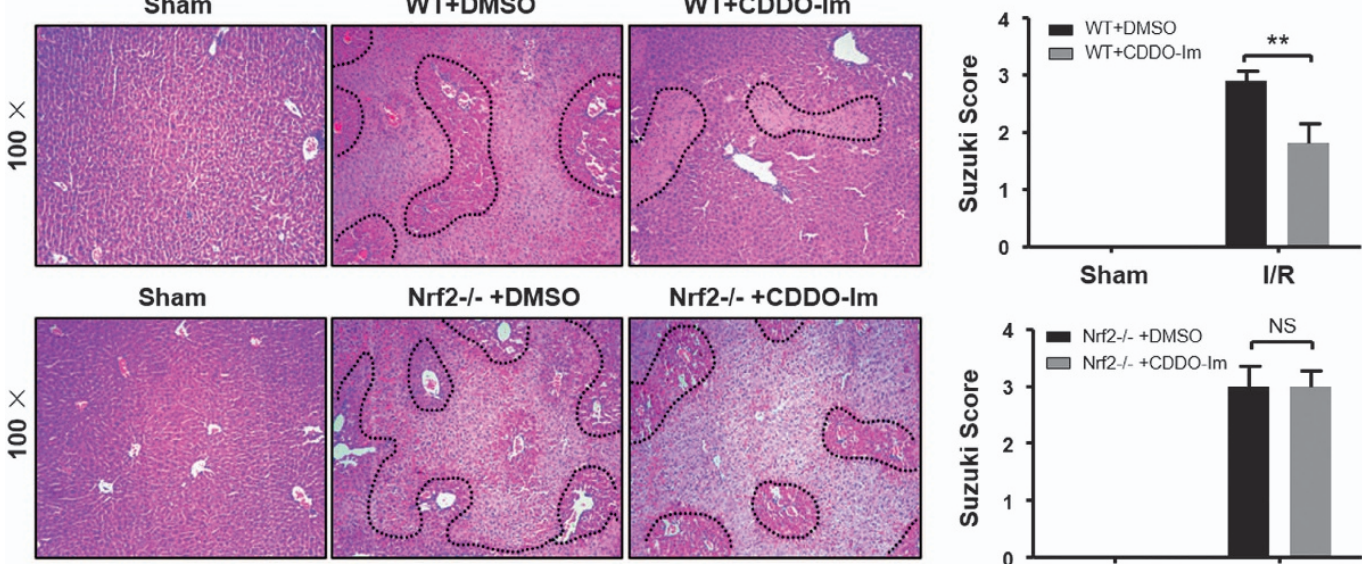

Nrf2-/- +CDDO-Im

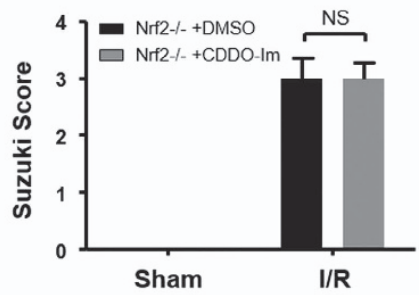

d

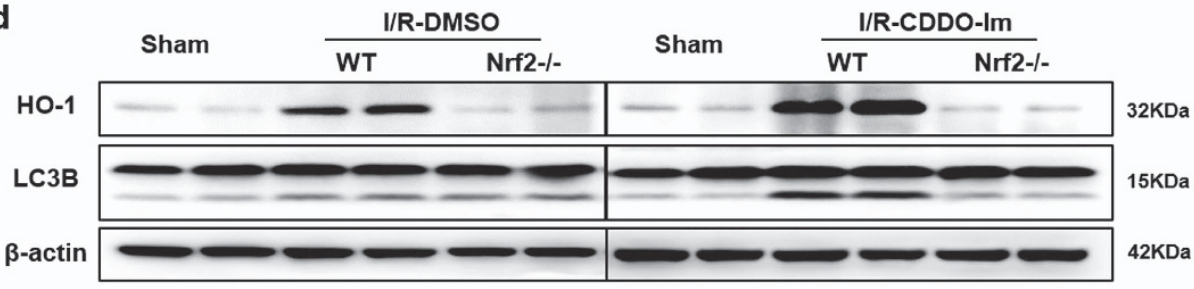

e

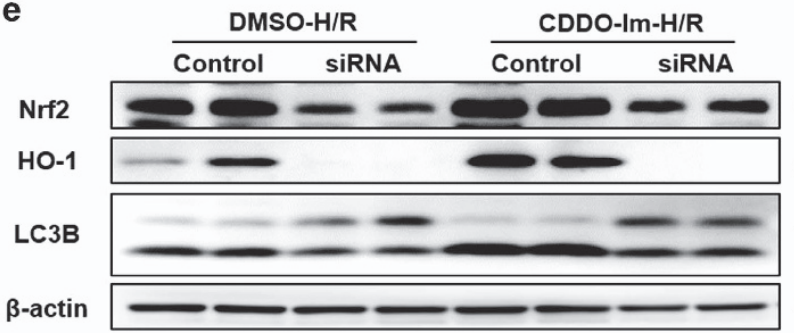

f $68 \mathrm{KDa}$ 32KDa $15 \mathrm{KDa}$ $42 \mathrm{KDa}$

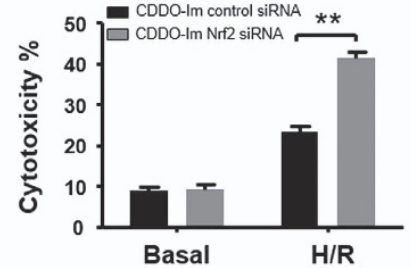


\#017009 from Jackson Lab, Bar Harbor, ME, USA) were kindly provided by Dr LiWei Dong, an associate investigator, in the Second Military Medical University, Shanghai, China. All procedures involving animals were reviewed and approved by the Institutional Animal Care and Use Committee of the Shanghai Jiao Tong University School of Medicine (approval no. SYKX-2008-0050).

Model of warm liver I/R injury. The model of partial hepatic I/R injury was used in our previous study. ${ }^{22}$ In brief, an atraumatic clip was used to interrupt the arterial and portal venous blood supply to the cephalad lobes of the liver for $90 \mathrm{~min}$. Sham controls underwent the same procedure, but without vascular occlusion. CDDO-Im (2 mg/kg) (Tocris Bioscience, Bristol, UK) was administered intraperitoneally $3 \mathrm{~h}$ before onset of liver ischemia, whereas chloroquine $(\mathrm{CQ}, 60 \mathrm{mg} / \mathrm{kg})$ or 3-Methyladenine (30 mg/kg) (Sigma-Aldrich, St. Louis, MO, USA) was administered intraperitoneally $1 \mathrm{~h}$ before the operation of liver ischemia. Tin Protoporphyrin IX dichlorid (SnPP) (50 mg/kg, Santa Cruz, Dallas, TX, USA) was administered intraperitoneally $1 \mathrm{~h}$ before injection of CDDO-Im or DMSO. In vehicle-treated mice, a volume of $0.5 \%$ DMSO or saline solution equal to that of treatment was administered in the same manner.

Biochemical measurement. Blood was collected by direct puncture of arteriae aorta then centrifuged at $3000 \times g$ for $5 \mathrm{~min}$. Serum ALT and AST levels were measured by standard clinical automatic analyzer (Dimension Xpand; Siemens Dade Behring, Munich, Germany). MDA was measured by microplate test kits (Nanjing Jiancheng Bioengineering Institute, Nanjing, China) according to the manufacturer's instructions.

Liver histopathology, IHC analyses and TUNEL staining. Liver tissues were fixed in $4 \%$ paraformaldehyde for at least $24 \mathrm{~h}$, then paraffin embedding through standard procedures and were cut into $5-\mu \mathrm{m}$-thick sections. For liver histopathology, the sections were stained with hematoxylin and eosin. Suzuki's criteria were used to evaluate the histological severity of liver injury. ${ }^{37}$ In brief, sinusoidal congestion, hepatocyte necrosis and ballooning degeneration were blindly graded from 0 to 4 . For IHC analyses, liver sections were first rehydrated and processed for an antigen-unmasking procedure, and then incubated with primary antibodies against MPO (Cell Signaling, Boston, MA, USA), cleaved caspase-3 (Cell Signaling), F4/80 (AbD Serotec, Kidlington, UK) and hemeoxygenase-1 (HO-1) (Cell Signaling) overnight at $4{ }^{\circ} \mathrm{C}$, followed by horseradish peroxidase-conjugated secondary antibodies. For histological analysis, sections were evaluated in a blinded manner by a pathologist. At least three fields per section were evaluated. TUNEL staining in liver sections was conducted by using an In-Situ Cell Death Detection Kit according to the manufacturer's instructions (Roche Diagnostics, Indianapolis, IN, USA). For each stained section, at least three images from random fields were taken, and at least three mice per group were subjected to each experiment. Image-Pro Plus 6.0 was used for image analysis of sections.

Hepatocyte isolation, culture and treatment. Primary hepatocyte isolation was performed as we previously described. ${ }^{38}$ The isolated cells were plated on dishes $\left(3 \times 10^{6} \mathrm{cells} / 6\right.$-cm dish), six-well plates $\left(2 \times 10^{5} \mathrm{celll} / \mathrm{well}\right)$ or 24-well plates $\left(8 \times 10^{4} \mathrm{cells} /\right.$ well). To simulate $\mathrm{H} / \mathrm{R}$ in vitro, hepatocytes were cultured for $4 \mathrm{~h}$ at $37{ }^{\circ} \mathrm{C}$ in a modular incubator chamber (Biospherix, Lacona, NY, USA) gassed with $5 \% \mathrm{CO}_{2}, 90 \% \mathrm{~N}_{2}$ and $5 \% \mathrm{O}_{2}$ for $4 \mathrm{~h}$. For reoxygenation, hepatocytes were returned to the normoxic incubator for $2 \mathrm{~h}$. To assess the cell injury, LDH was measured by LDH Release Assay Kit (Beyotime, Shanghai, China) according to the manufacturer's protocols. Mitochondrial transmembrane potential in hepatocytes was assessed by $\Delta \Psi \mathrm{m}$ assay kit with JC-1 (Beyotime) according to the manufacturer's protocols. The decrease of $\Delta \Psi \mathrm{m}$ was assessed by transition from JC-1 aggregates (Red) to JC-1 monomer (Green) in cells which indicating mitochondrial dysfunction and early cell death. Mitochondrial generation of superoxide was stained with MitoSOX Red (Invitrogen, Waltham, MA, USA). The Cyto-ID Autophagy Detection Kit (Enzo Life Sciences, Farmington, NY, USA) was used to identify the autophagosomes of the hepatocytes. In brief, after $H / R$ injury, the hepatocytes were incubated in Cyto-ID Green stain solution in the dark for $30 \mathrm{~min}$ at $37^{\circ} \mathrm{C}$. Then analyze the stained cells by fluorescence microscope.

Quantitative RT-PCR. Total liver RNA was extracted using TRIzol (Takara, Tokyo, Japan) reagent according to the manufacturer's instructions. The CDNA was synthesized using $1000 \mathrm{ng}$ of total RNA in the first-strand cDNA synthesis reaction with PrimeScript RT reagent Kit (Takara). RT-PCR was performed using the CFX 96 q-PCR system (BIO-RAD, Hercules, CA, USA). A SYBR RT-PCR kit (Takara) were used for quantitative real-time RT-PCR analysis. All reactions were conducted in a $20 \mu \mathrm{l}$ reaction volume in triplicate. The relative expression levels for a target gene were normalized by $\beta$-actin. Primers used for RT-PCR analysis are:

Gclc forward: 5'- ATGTGGACACCCGATGCAGTATT

Gclc reverse: 5'-TGTCTTGCTTGTAGTCAGGATGGTTT

Gclm forward: 5'- TGGAGCAGCTGTATCAGTGG

Gclm reverse: 5' - AGAGCAGTTCTTTCGGGTCA

Gstm1 forward: 5'- CTACCTTGCCCGAAAGCAC

Gstm1 reverse: 5'- ATGTCTGCACGGATCCTCT

Nq01 forward: 5'- AGCGTTCGGTATTACGATCC

Nq01 reverse: $5^{\prime}$ - AGTACAATCAGGGCTCTTCTCG

$111 \mathrm{~b}$ forward: $5^{\prime}$ - TGTAATGAAAGACGGCACACC

$1 / 1 b$ reverse: $5^{\prime}$ - TCTTCTTTGGGTATTGCTTGG

Tnf forward: 5'- TTCTATGGCCCAGACCCTCA

Tnf reverse: 5'- TTTGCTACGACGTGGGCTAC

116 forward: 5'- GCTACCAAACTGGATATAATCAGGA

II6 reverse: $5^{\prime}$ - CCAGGTAGCTATGGTACTCCAGAA

CXCl-10 forward: 5'- GCTGCCGTCATTTTCTGC

CXCl-10 reverse: 5' - TCTCACTGGCCCGTCATC

Actb forward: 5'- TGACAGGATGCAGAAGGAGA

Actb reverse: 5' - ACCGATCCACACAGAGTACT.

Western blots. Liver tissues were processed to western blot analysis as we previously described. ${ }^{38}$ Nuclear extracts were isolated from the livers or cultured hepatocytes, using NE-PER Nuclear and Cytoplasmic Extraction Reagents (Thermo Fisher scientific, Waltham, MA, USA). The primary antibodies against $\mathrm{Bcl} 2, \mathrm{Bcl}-\mathrm{XL}$, cleaved caspase-3, HO-1, LC3B, SQSTM1 (Cell Signaling), Nrf2 (Abcam), $\beta$-actin (Sigma-Aldrich) were used.

TEM analysis. The liver tissues were fixed with $2.5 \%$ glutaraldehyde Ultrathin sections were cut and doubly stained with uranyl acetate and lead citrate. For autophagic vacuole quantification, 10 micrographs, primary magnification $\times 15000$, were randomly taken from each sample and the total amount of autophagic vacuoles was counted.

Enzyme-linked immunosorbent assay (ELISA). ELISA kits were used to detect mouse serum IL-6, TNF $\alpha$ (NeoBioscience Technology, Shenzhen, China) according to the manufacturer's protocols.

Caspase- 3 activities. Caspase- 3 activities were detected in liver tissues. The activities were measured with caspase-3 assay kit (Jiancheng Biotechnology, Nanjing, China) according to the manufacturer's instructions.

RNA interference in primary hepatocytes. Double-stranded siRNA corresponding to homologous sequence of mouse Nrf2 gene (Santa Cruz

Figure 7 CDDO-Im in induction of autophagy depends on induction of Nrf2/HO-1 pathway. (a) Western blot analysis of nuclear and cytoplasm Nrf2 protein expression both in the CDDO-Im and DMSO pretreatment mice livers at $6 \mathrm{~h}$ after reperfusion and primary hepatocytes isolated from WT, which subjected to H/R injury. TBP served as a nuclear loading control, whereas $\beta$-actin served as a cytoplasm loading control. (b) WT and Nrf2 KO mice were pretreated with CDDO-Im (2 mg/kg, IP) or DMSO and killed at $6 \mathrm{~h}$ after reperfusion. (b) Serum ALT and AST levels. (c) Representative hematoxylin and eosin(HE) stained sections (original magnification, $\times 100$ ) and relevant average Suzuki score. (d) Western blot analysis of HO-1 and LC3B both in the CDDO-Im and DMSO pretreatment WTand Nrf2 KO mice livers at $6 \mathrm{~h}$ after reperfusion. $(n=3-4$ per group, mean \pm S.E.M., ${ }^{\star \star} P<0.01,{ }^{*} P<0.05$ ). (e) Immunoblots indicating expression of Nrf2, HO-1 and LC3B protein after treatment with Nrf2 siRNA in the presence and absence of CDDO-Im $(200 \mu \mathrm{M})$. (f) Quantification of average cytotoxicity (\% cell death) in different groups were plotted. Three to five replicates per experiment group, mean \pm S.E.M., ${ }^{\star \star} P<0.01$, ${ }^{*} P<0.05$ 

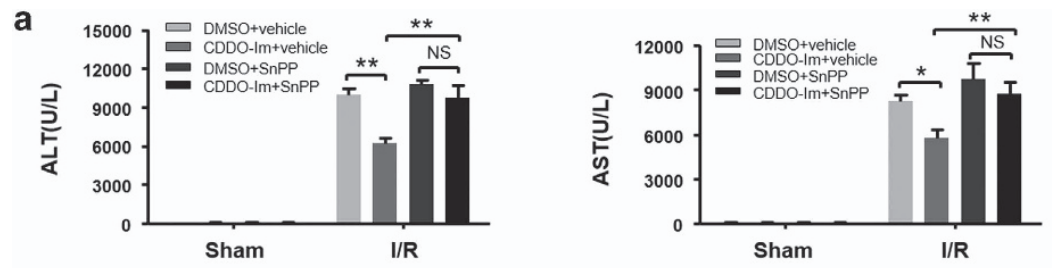

b
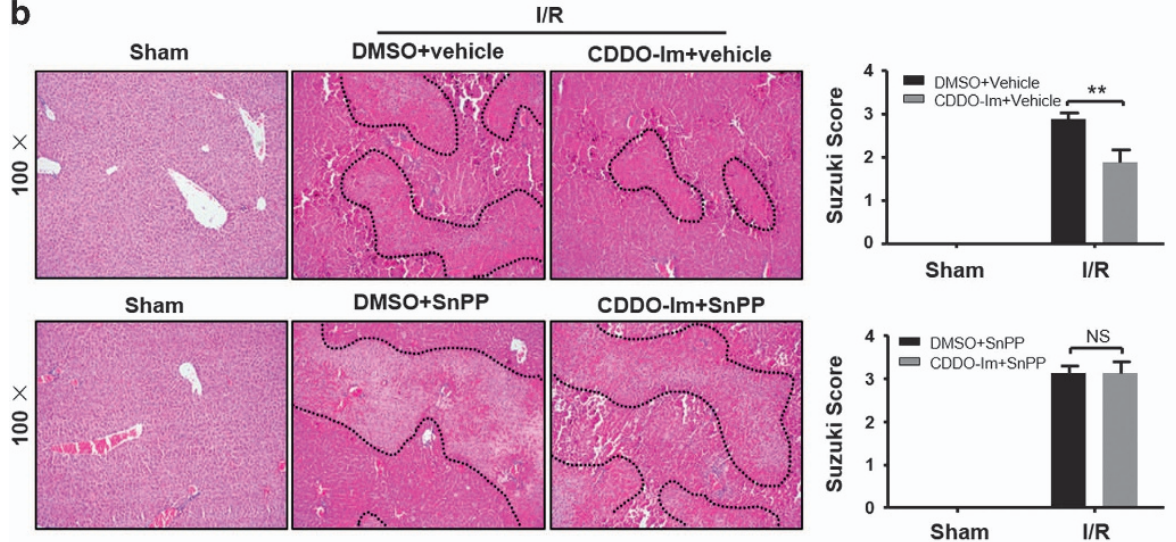

c

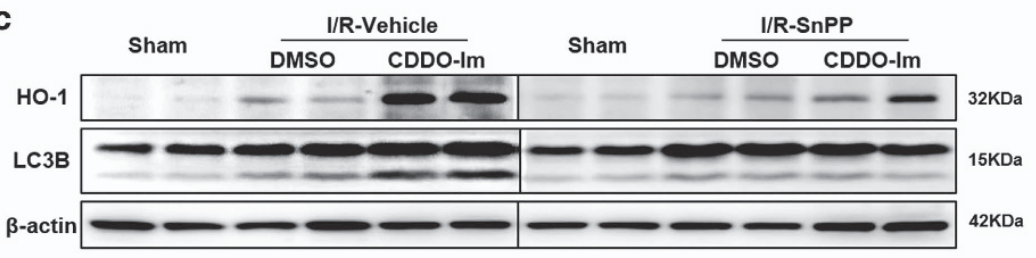

d
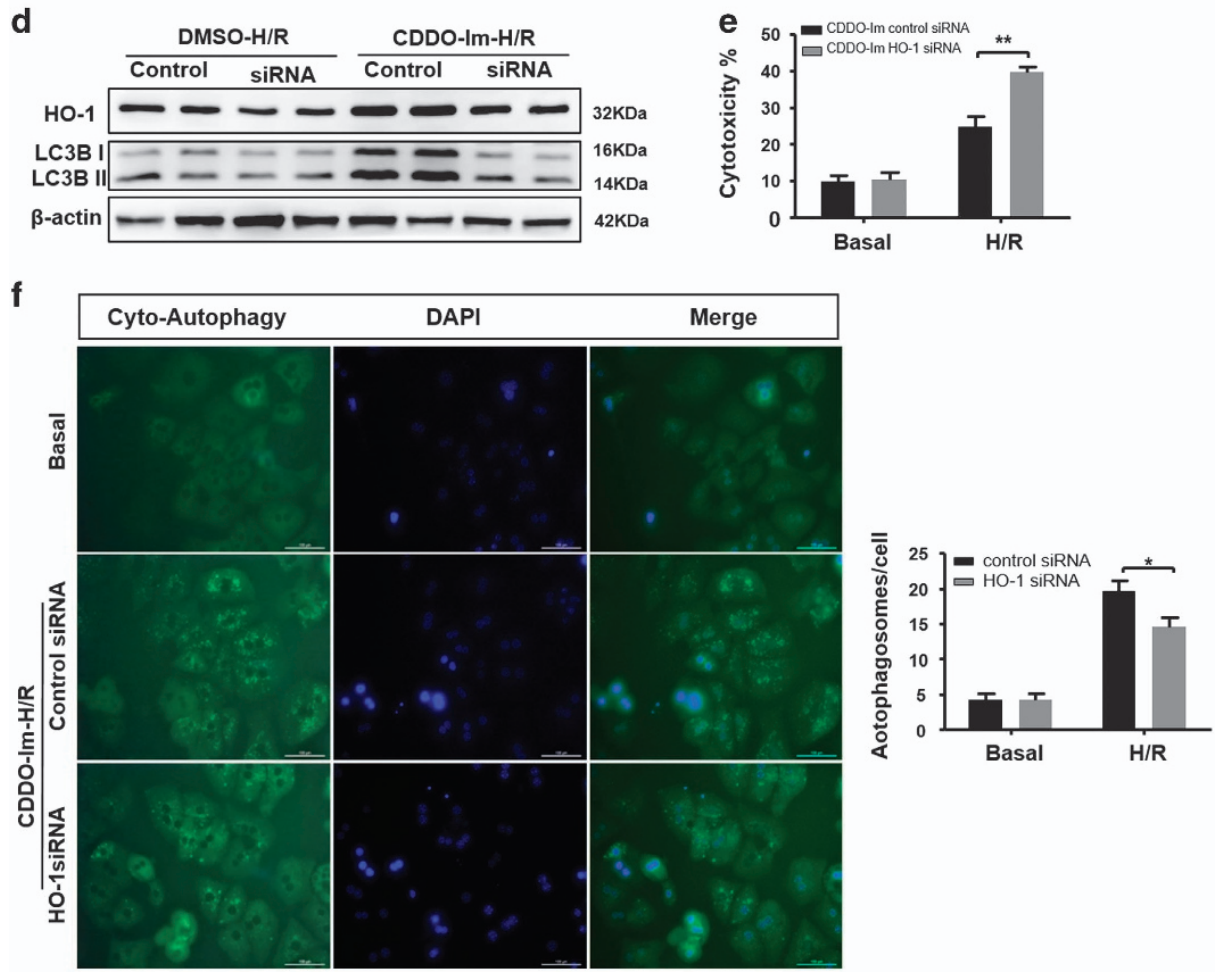
Figure 8 CDDO-Im in induction of autophagy depends on induction of HO-1. (a-c) WT mice were pretreated with SnPP (50 mg/kg, IP) $1 \mathrm{~h} \mathrm{before} \mathrm{CDDO-Im} \mathrm{(2} \mathrm{mg/kg,} \mathrm{IP)} \mathrm{or}$ DMSO and killed at $6 \mathrm{~h}$ after reperfusion. (a) Serum ALT and AST levels. (b) Representative hematoxylin and eosin (HE) stained sections (original magnification, $\times 100)$ and relevant average Suzuki score. (c) Western blot analysis of HO-1 and LC3B both in the SnPP and vehicle pretreatment with or without CDDO-Im mice livers at $6 \mathrm{~h}$ after reperfusion. ( $n=3-4$ per group, mean \pm S.E.M., ${ }^{* \star} P<0.01,{ }^{*} P<0.05$ ). (d) Immunoblots indicating expression of HO-1 and LC3B protein after treatment with HO-1 siRNA in the presence and absence of CDDO-Im (200 $\mu \mathrm{M})$ and densitometric analysis of LC3B-II and HO-1 expression. (e) Quantification of average cytotoxicity (\% cell death) in different group were plotted. (f) Representative fluorescence micrographs display autophagosomes in primary hepatocytes with CDDO-Im in the presence HO-1 siRNA or control siRNA (original magnification, $\times 400)$. The numbers of autophagosomes were determined. $\left(n=3-4\right.$ per group, mean \pm S.E.M., $\left.{ }^{* *} P<0.01,{ }^{*} P<0.05\right)$

Biotechnology, Dallas, TX, USA) and HO-1 gene (Gene Pharma, Shanghai, China) were used to inhibit Nrf2 and HO-1 expression. Transfection was conducted by using Lipofectamine RNAiMAX reagent (Life Technologies, Co., Grand Island, NY, USA) following the manufacturer's instructions. A scrambled siRNA was transfected as the negative control. After transfection for $48 \mathrm{~h}$, primary hepatocytes were followed by different drugs treatment and $H / R$ injury for further study.

mtDNA detection. mtDNA was measured by absolute quantitative real-time PCR as describe. ${ }^{39}$ Total DNA was isolated from plasma samples using QIAamp Blood and Mini Kit (Qiagen, Valencia, CA, USA), which removes plasma components with the potential to interfere in PCR analysis. The samples were then diluted, and the same amount of total DNA was added to each reaction on each plate. The amount of mtDNA was determined using a pair of primers targeting mouse cytochrome $c$ oxidase subunit III (Forward: 5'-ACCAAGGCCACCACA CTCCT-3' and Reverse: 5'-ACGCTCAGAAGAATCCTGCAAAGAA-3'). To construct standard curves, mitochondrial pellets were isolated from mouse liver by differential centrifugation. Purity of mtDNA standards was verified by real-time PCR using primers for both mitochondrial genes and nuclear-encoded $\beta$-actin. Dilutions of these purified mtDNA samples were prepared and standards were included on each PCR plate for each gene tested. The limit of detection for the assay was determined to be $<0.05 \mathrm{ng} / \mathrm{ml}$.

Statistical analysis. Data were expressed as mean \pm S.E.M. Statistical significance was determined by two tailed, unpaired or paired Student's $t$-test. A $P$-value $<0.05$ was used to indicate a statistically significant difference in all statistical comparisons,

\section{Conflict of Interest}

The authors declare no conflict of interest.

Acknowledgements. This work was supported by the National Natural Science Foundation of China (81670562 and 31300742 to X Kong, 81372233 to $\mathrm{H} \mathrm{Wu}, 81670598$ to Q Xia, 81670523 to X Chen), and grant from the Committee of Science and Technology of Shanghai Municipal Government (16401970600-03) to X Kong, the Shanghai Health Bureau Key Joint Efforts Foundation (2013ZYJB001) to $Q$ Xia, The Doctoral Innovation Fund Projects of Shanghai Jiao Tong University School of Medicine (BXJ201719) to D Xu.

\section{Publisher's Note}

Springer Nature remains neutral with regard to jurisdictional claims in published maps and institutional affiliations.

1. Khandoga A, Hanschen M, Kessler JS, Krombach F. CD4+ T cells contribute to postischemic liver injury in mice by interacting with sinusoidal endothelium and platelets. Hepatology (Baltimore, Md) 2006; 43: 306-315.

2. Abe $Y$, Hines I, Zibari G, Grisham MB. Hepatocellular protection by nitric oxide or nitrite in ischemia and reperfusion injury. Arch Biochem Biophys 2009; 484: 232-237.

3. Zhai Y, Petrowsky H, Hong JC, Busuttil RW, Kupiec-Weglinski JW. Ischaemia-reperfusion injury in liver transplantation-from bench to bedside. Nat Rev Gastroenterol Hepatol 2013; 10: $79-89$.

4. Kensler TW, Wakabayashi N, Biswal S. Cell survival responses to environmental stresses via the Keap1-Nrf2-ARE pathway. Ann Rev Pharmacol Toxicol 2007; 47: 89-116.

5. Kong X, Thimmulappa R, Craciun F, Harvey C, Singh A, Kombairaju P et al. Enhancing Nrf2 pathway by disruption of Keap1 in myeloid leukocytes protects against sepsis. Am J Respir Crit Care Med 2011; 184: 928-938.

6. Kong X, Thimmulappa R, Kombairaju P, Biswal S. NADPH oxidase-dependent reactive oxygen species mediate amplified TLR4 signaling and sepsis-induced mortality in Nrf2-deficient mice. J Immunol 2010; 185: 569-577.

7. Ke B, Shen XD, Zhang Y, Ji H, Gao F, Yue $S$ et al. KEAP1-NRF2 complex in ischemiainduced hepatocellular damage of mouse liver transplants. J Hepatoly 2013; 59: 1200-1207.
8. Osburn WO, Yates MS, Dolan PD, Chen S, Liby KT, Sporn MB et al. Genetic or pharmacologic amplification of nrf2 signaling inhibits acute inflammatory liver injury in mice. Toxicol Sci 2008; 104: 218-227.

9. Reisman SA, Yeager RL, Yamamoto M, Klaassen CD. Increased Nrf2 activation in livers from Keap1-knockdown mice increases expression of cytoprotective genes that detoxify electrophiles more than those that detoxify reactive oxygen species. Toxicol Sci 2009; 108: 35-47.

10. Reisman SA, Ward KW, Klaassen CD, Meyer CJ. CDDO-9,11-dihydro-trifluoroethyl amide (CDDO-dhTFEA) induces hepatic cytoprotective genes and increases bile flow in rats. Xenobiotica 2013; 43: 571-578.

11. Copple BL, Jaeschke H, Klaassen CD. Oxidative stress and the pathogenesis of cholestasis. Semin Liver Dis 2010; 30: 195-204.

12. Thimmulappa RK, Fuchs RJ, Malhotra D, Scollick C, Traore K, Bream JH et al. Preclinical evaluation of targeting the Nrf2 pathway by triterpenoids (CDDO-Im and CDDO-Me) for protection from LPS-induced inflammatory response and reactive oxygen species in human peripheral blood mononuclear cells and neutrophils. Antioxid Redox Signal 2007; 9: 1963-1970.

13. Reddy NM, Suryanaraya V, Yates MS, Kleeberger SR, Hassoun PM, Yamamoto M et al. The triterpenoid CDDO-imidazolide confers potent protection against hyperoxic acute lung injury in mice. Am J Respir Crit Care Med 2009; 180: 867-874.

14. Zhang F, Wang S, Zhang M, Weng Z, Li P, Gan Y et al. Pharmacological induction of heme oxygenase- 1 by a triterpenoid protects neurons against ischemic injury. Stroke 2012; 43 : 1390-1397.

15. Liu M, Reddy NM, Higbee EM, Potteti HR, Noel S, Racusen L et al. The Nrf2 triterpenoid activator, CDDO-imidazolide, protects kidneys from ischemia-reperfusion injury in mice. Kidney Int 2014; 85: 134-141.

16. Sussan TE, Rangasamy T, Blake DJ, Malhotra D, El-Haddad H, Bedja D et al. Targeting Nrf2 with the triterpenoid CDDO-imidazolide attenuates cigarette smoke-induced emphysema and cardiac dysfunction in mice. Proc Natl Acad Sci USA 2009; 106: 250-255.

17. Reisman SA, Buckley DB, Tanaka Y, Klaassen CD. CDDO-Im protects from acetaminophen hepatotoxicity through induction of Nrf2-dependent genes. Toxicol Appl Pharmacol 2009; 236: 109-114.

18. Yorimitsu T, Klionsky DJ. Autophagy: molecular machinery for self-eating. Cell Death Differ 2005; 12: 1542-1552.

19. Rautou PE, Mansouri A, Lebrec D, Durand F, Valla D, Moreau R. Autophagy in liver diseases. J Hepatol 2010; 53: 1123-1134.

20. Murrow L, Debnath J. Autophagy as a stress-response and quality-control mechanism: implications for cell injury and human disease. Ann Rev Pathol 2013; 8: 105-137.

21. Gottlieb RA, Mentzer RM. Autophagy during cardiac stress: joys and frustrations of autophagy. Ann Rev Physiol 2010; 72: 45-59.

22. Zhu J, Lu T, Yue S, Shen X, Gao F, Busuttil RW et al. Rapamycin protection of livers from ischemia and reperfusion injury is dependent on both autophagy induction and mammalian target of rapamycin complex 2-Akt activation. Transplantation 2015; 99: 48-55.

23. Schwabe RF, Brenner DA. Mechanisms of Liver Injury. I. TNF-alpha-induced liver injury: role of IKK, JNK, and ROS pathways. Am J Physiol Gastrointest Liver Physiol 2006; 290: G583-G589.

24. Wang AL, Niu Q, Shi N, Wang J, Jia XF, Lian HF et al. Glutamine ameliorates intestinal ischemia-reperfusion Injury in rats by activating the Nrf2/Are signaling pathway. Int $J$ Clin Exp Pathol 2015; 8: 7896-7904.

25. Huang Q, Zhan L, Cao H, Li J, Lyu Y, Guo X et al. Increased mitochondrial fission promotes autophagy and hepatocellular carcinoma cell survival through the ROS-modulated coordinated regulation of the NFKB and TP53 pathways. Autophagy 2016; 12: 999-1014.

26. Liby K, Hock T, Yore MM, Suh N, Place AE, Risingsong R et al. The synthetic triterpenoids, CDDO and CDDO-imidazolide, are potent inducers of heme oxygenase-1 and Nrf2/ARE signaling. Cancer Res 2005; 65: 4789-4798.

27. Liu A, Fang H, Wei W, Dirsch O, Dahmen U. Ischemic preconditioning protects against liver ischemia/reperfusion injury via heme oxygenase-1-mediated autophagy. Criti Care Med 2014; 42: e762-e771.

28. Yun N, Cho HI, Lee SM. Impaired autophagy contributes to hepatocellular damage during ischemia/reperfusion: heme oxygenase-1 as a possible regulator. Free Radic Biol Med 2014; 68: 168-177

29. Czaja MJ, Ding WX, Donohue TM Jr., Friedman SL, Kim JS, Komatsu M et al. Functions of autophagy in normal and diseased liver. Autophagy 2013; 9: 1131-1158.

30. Kim JS, Nitta T, Mohuczy D, O'Malley KA, Moldawer LL, Dunn WA Jr. et al. Impaired autophagy: A mechanism of mitochondrial dysfunction in anoxic rat hepatocytes. Hepatology (Baltimore, Md) 2008; 47: 1725-1736.

31. Wang JH, Ahn IS, Fischer TD, Byeon Jl, Dunn WA Jr., Behrns KE et al. Autophagy suppresses age-dependent ischemia and reperfusion injury in livers of mice. Gastroenterology 2011; 141: 2188-2199.e2186. 
32. Khader A, Yang WL, Godwin A, Prince JM, Nicastro JM, Coppa GF et al. Sirtuin 1 stimulation attenuates ischemic liver injury and enhances mitochondrial recovery and autophagy. Criti Care Med 2016; 44: e651-e663.

33. Lee SC, Kim KH, Kim OH, Lee SK, Kim SJ. Activation of autophagy by everolimus confers hepatoprotection against ischemia-reperfusion injury. Am J Transplant 2016; 16: 2042-2054.

34. Bao LJ, Jaramillo MC, Zhang ZB, Zheng YX, Yao M, Zhang DD et al. Nrf2 induces cisplatin resistance through activation of autophagy in ovarian carcinoma. Int J Clin Exp Pathol 2014; 7: $1502-1513$

35. Carchman EH, Rao J, Loughran PA, Rosengart MR, Zuckerbraun BS. Heme oxygenase-1mediated autophagy protects against hepatocyte cell death and hepatic injury from infection/ sepsis in mice. Hepatology (Baltimore, Md) 2011; 53: 2053-2062.

36. Lee SJ, Ryter SW, Xu JF, Nakahira K, Kim HP, Choi AM et al. Carbon monoxide activates autophagy via mitochondrial reactive oxygen species formation. Am J Respir Cell Mol Biol 2011; 45: 867-873.

37. Suzuki S, Toledo-Pereyra LH, Rodriguez FJ, Cejalvo D. Neutrophil infiltration as an important factor in liver ischemia and reperfusion injury. Modulating effects of FK506 and cyclosporine. Transplantation 1993; 55: 1265-1272.

38. Wen Y, Feng D, Wu H, Liu W, Li H, Wang F et al. Defective initiation of liver regeneration in osteopontin-deficient mice after partial hepatectomy due to insufficient activation of IL-6/Stat3 pathway. Int J Biol Sci 2015; 11: 1236-1247.
39. McGill MR, Sharpe MR, Williams CD, Taha M, Curry SC, Jaeschke H. The mechanism underlying acetaminophen-induced hepatotoxicity in humans and mice involves mitochondrial damage and nuclear DNA fragmentation. J Clin Invest 2012; 122: 1574-1583.

cc) (i) Cell Death and Disease is an open-access journal published by Nature Publishing Group. This work is licensed under a Creative Commons Attribution 4.0 International License. The images or other third party material in this article are included in the article's Creative Commons license, unless indicated otherwise in the credit line; if the material is not included under the Creative Commons license, users will need to obtain permission from the license holder to reproduce the material. To view a copy of this license, visit http://creativecommons.org/licenses/by/4.0/

(C) The Author(s) 2017

Supplementary Information accompanies this paper on Cell Death and Disease website (http://www.nature.com/cddis) 